\title{
Scale dependence and cross-scale transfer of kinetic energy in compressible hydrodynamic turbulence at moderate Reynolds numbers
}

\author{
Petr Hellinger $\odot,{ }^{1,2,{ }^{*}}$ Andrea Verdini $\odot,{ }^{3,4}$ Simone Landi $\odot,{ }^{3,4}$ Emanuele Papini $\odot,{ }^{3,4}$ \\ Luca Franci $\odot, 5,4$ and Lorenzo Matteini $\oplus^{6,4}$ \\ ${ }^{1}$ Astronomical Institute, CAS, Bocni II/1401, CZ-14100 Prague, Czech Republic \\ ${ }^{2}$ Institute of Atmospheric Physics, CAS, Bocni II/1401, CZ-14100 Prague, Czech Republic \\ ${ }^{3}$ Dipartimento di Fisica e Astronomia, Università degli Studi di Firenze Largo E. Fermi 2, \\ I-50125 Firenze, Italy \\ ${ }^{4}$ INAF-Osservatorio Astrofisico di Arcetri, Largo E. Fermi 5, I-50125 Firenze, Italy \\ ${ }^{5}$ School of Physics and Astronomy, Queen Mary University of London, London E1 4NS, United Kingdom \\ ${ }^{6}$ Department of Physics, Imperial College London, London SW7 2AZ, United Kingdom
}

(Received 28 October 2020; accepted 18 March 2021; published 13 April 2021)

We investigate the properties of the scale dependence and cross-scale transfer of kinetic energy in compressible three-dimensional hydrodynamic turbulence by means of two direct numerical simulations of decaying turbulence with initial Mach numbers $M=1 / 3$ and 1 , and with moderate Reynolds numbers, $R_{\lambda} \sim 100$. The turbulent dynamics is analyzed using compressible and incompressible versions of the dynamic spectral transfer (ST) and the Kármán-Howarth-Monin (KHM) equations. We find that the nonlinear coupling leads to a flux of the kinetic energy to small scales where it is dissipated; at the same time, the reversible pressure-dilatation mechanism causes oscillatory exchanges between the kinetic and internal energies with an average zero net energy transfer. While the incompressible KHM and ST equations are not generally valid in the simulations, their compressible counterparts are well satisfied and describe, in a quantitatively similar way, the decay of the kinetic energy on large scales, the cross-scale energy transfer/cascade, the pressure dilatation, and the dissipation. There exists a simple relationship between the KHM and ST results through the inverse proportionality between the wave vector $k$ and the spatial separation length $l$ as $k l \simeq \sqrt{3}$. For a given time, the dissipation and pressure-dilatation terms are strong on large scales in the KHM approach, whereas the ST terms become dominant on small scales; this is due to the complementary cumulative behavior of the two methods. The effect of pressure dilatation is weak when averaged over a period of its oscillations and may lead to a transfer of the kinetic energy from large to small scales without a net exchange between the kinetic and internal energies. Our results suggest that for large-enough systems, there exists an inertial range for the kinetic energy cascade. This transfer is partly due to the classical, nonlinear advection-driven cascade and partly due to the pressure dilatation-induced energy transfer. We also use the ST and KHM approaches to investigate the properties of the internal energy. The dynamic ST and KHM equations for the internal energy are well satisfied in the simulations but behave very differently with respect to the viscous dissipation. We conclude that ST and KHM approaches would better be used for the kinetic and internal energies separately.

DOI: 10.1103/PhysRevFluids.6.044607

*petr.hellinger@asu.cas.cz 


\section{INTRODUCTION}

The fundamental problems of turbulence concern how the energy (and other quantities) is distributed on spatiotemporal scales, how it is transferred across scales, and how it is exchanged among its different forms. The current understanding of turbulence is mostly based on the hydrodynamic model in the incompressible limit [1], where the divergence of the velocity field is taken to be zero, and a constant density is usually assumed. In this case, the spatial-scale decomposition of the kinetic energy (per mass) may be characterized by the spectral density of the velocity field [2,3], and its evolution can be analyzed using spectral transfer (ST) approaches [4,5]. Alternatively, one can look at the cross-correlations of the velocity field or structure functions (related to the power spectrum) via the Kármán-Howarth-Monin (KHM) equation [6-8]. Another possibility is to use space-filtering (coarse graining) of the velocity field $[9,10]$. These approaches may be used to quantitatively characterize the different turbulence processes, the injection/decay, the cross-scale energy transfer, and the dissipation. Moreover, they can be used to determine whether an inertial range exists, where the only relevant process is the cross-scale energy transfer, and if this transfer is cascade-like [11], i.e., if the cross-scale energy transfer is dominated by interactions between nearby scales.

Extension of the incompressible results to the case of general, compressible fluids with variable densities is not trivial [12-14]. It is not evident how to characterize, in an analogous manner, the scale distribution of the kinetic energy when the density is not constant [15-18]. There are multiple different density weighting methods for the spectral, structure function, and coarse-graining approaches. Furthermore, the compressibility introduces the pressure-dilatation effect that couples the kinetic and internal energies in a reversible manner (in contrast to the irreversible viscous dissipation). The pressure-dilatation channel brings into question the existence of an inertial range for the kinetic energy.

Numerical simulation results of Refs. [12,19] indicate that the pressure-dilatation induced energy exchanges tend to be more important on large scales. Reference [19] shows that the strength of the pressure-dilatation effect decreases on a small scale so that there can exist a range of scales where the pressure dilatation is negligible and the kinetic energy conservatively cascades. On the other hand, Ref. [20] shows that the pressure dilatation appears on small scales (and may lead to cross-scale transfer of the kinetic energy).

Since the kinetic and internal energies are coupled via the dissipation, as well as through the pressure dilatation, one may consider the total (kinetic+internal) energy, which is strictly conserved. References [21,22] formulate the KHM equation in the compressible case for the total energy. They assume, however, that the system follows a given closure (isothermal or polytropic), and they use the closure to derive the KHM equation. In particular, they manipulate the pressure-dilatation term to cast it in a form of a cascade rate; it is unclear if all or only a part of the pressure-dilatation effects are present in such a system.

Here we address the pressure-dilatation effect, its role in the compressible hydrodynamic (HD) turbulence, and its characteristic scales using two methods. We reexamine the KHM equation for the kinetic energy in compressible HD, and we analyze the results of direct compressible HD numerical simulations. We compare these results with those of a simple ST approach in both the incompressible and compressible approximations. We also look at the properties of the internal energy and its scale decomposition, and we compare these results with those of the kinetic energy. The paper is organized as follows: In Sec. II we present an overview of two direct three-dimensional (3D) HD simulations. In Sec. III we present the ST Fourier method, and we use it to analyze the simulation results. In Sec. IV we rederive the KHM equation for the kinetic energy, and we apply it to the simulation results; the results of the two methods in both incompressible and compressible approximations are compared. In Sec. V we test the scale decomposition of the internal energy using the ST and KHM approaches. Finally, in Sec. VI we discuss the obtained results. 
TABLE I. Simulation parameters.

\begin{tabular}{lcccccc}
\hline \hline Run & Grid & Size & $M$ & $\mu$ & $t_{\omega}$ & $R_{\lambda}$ \\
\hline 1 & $1024^{3}$ & $(2 \pi)^{3}$ & $1 / 3$ & $4 \times 10^{-4}$ & 6.6 & 146 \\
2 & $1024^{3}$ & $(2 \pi)^{3}$ & 1 & $2 \times 10^{-3}$ & 6.5 & 82 \\
\hline \hline
\end{tabular}

\section{NUMERICAL SIMULATIONS}

We employ a 3D pseudospectral compressible hydrodynamic code derived from the compressible magnetohydrodynamic (MHD) code [23] based on the P3DFFT library [24] and FFTW3 [25]. The code solves the compressible Navier-Stokes equations for the fluid density $\rho$, velocity $\boldsymbol{u}$, and the pressure $p$ :

$$
\begin{gathered}
\frac{\partial \rho}{\partial t}+\nabla \cdot(\rho \boldsymbol{u})=0, \\
\frac{\partial(\rho \boldsymbol{u})}{\partial t}+\nabla \cdot(\rho \boldsymbol{u} \boldsymbol{u})=-\nabla p+\nabla \cdot \boldsymbol{\tau},
\end{gathered}
$$

complemented with an equation for the temperature $T=p / \rho$,

$$
\frac{\partial T}{\partial t}+(\boldsymbol{u} \cdot \nabla) T=\frac{\alpha}{\rho} \Delta T+\frac{\gamma-1}{\rho}(-p \theta+\Sigma: \boldsymbol{\tau}),
$$

where $\theta=\boldsymbol{\nabla} \cdot \boldsymbol{u}$ is the dilatation, $\boldsymbol{\Sigma}=\boldsymbol{\nabla} \boldsymbol{u}$ is the stress tensor, and $\boldsymbol{\tau}$ is the viscous stress tensor $\left[\tau_{i j}=\mu\left(\partial u_{i} / \partial x_{j}+\partial u_{j} / \partial x_{i}-2 / 3 \theta \delta_{i j}\right)\right.$; here the dynamic viscosity $\mu$ is assumed to be constant $]$, and $\alpha$ is the thermal diffusivity (we set $\alpha=\mu$ and $\gamma=5 / 3$ ). The colon operator denotes the double contraction of second-order tensors, $\mathbf{A}: \mathbf{B}=\sum_{i j} A_{i j} B_{i j}$.

For the compressible Navier-Stokes equations (2), one gets the following equation for the average kinetic energy in a closed system:

$$
\frac{\partial}{\partial t}\left\langle\frac{1}{2} \rho|\boldsymbol{u}|^{2}\right\rangle=\langle p \theta\rangle-\langle\boldsymbol{\tau}: \boldsymbol{\Sigma}\rangle,
$$

where $\langle\bullet\rangle$ denotes spatial averaging over the domain (the simulation box). The two terms on the right-hand side of Eq. (4) couple the kinetic energy to the internal one,

$$
\frac{\partial}{\partial t}\langle\rho e\rangle=-\langle p \theta\rangle+\langle\boldsymbol{\tau}: \boldsymbol{\Sigma}\rangle
$$

where $e=T /(\gamma-1)$ is the internal energy density (per mass).

We perform two simulations of decaying turbulence with different levels of compressibility. The simulation box size is $(2 \pi)^{3}$ (with a grid of $1024^{3}$ points), and periodic boundary conditions are assumed. Both simulations are initialized with isotropic, random-phase, solenoidal fluctuations (i.e., $\theta$ is set to 0 ) on large scales (with wave-vector magnitudes $k=|\boldsymbol{k}| \leqslant 4$ ). Run 1 starts with the rms Mach number $M=1 / 3$, whereas for run 2 we set the initial Mach number $M=1$. For run 1 we set the (constant) dynamic viscosity $\mu=4 \times 10^{-4}$, and for run 2 we set $\mu=2 \times 10^{-3}$; we use a large viscosity in this case to avoid steep gradients (shocks) that are not well resolved by pseudospectral codes. Table I gives an overview of the simulation parameters. Table I also shows the times $t_{\omega}$ where the rms of the vorticity reaches the maximum and the microscale Reynolds number, $R_{\lambda}$, given by [26]

$$
R_{\lambda}=\left(\frac{5}{3\langle\boldsymbol{\tau}: \Sigma\rangle}\right)^{1 / 2}\langle\rho\rangle\left\langle|\boldsymbol{u}|^{2}\right\rangle
$$

at that time. 


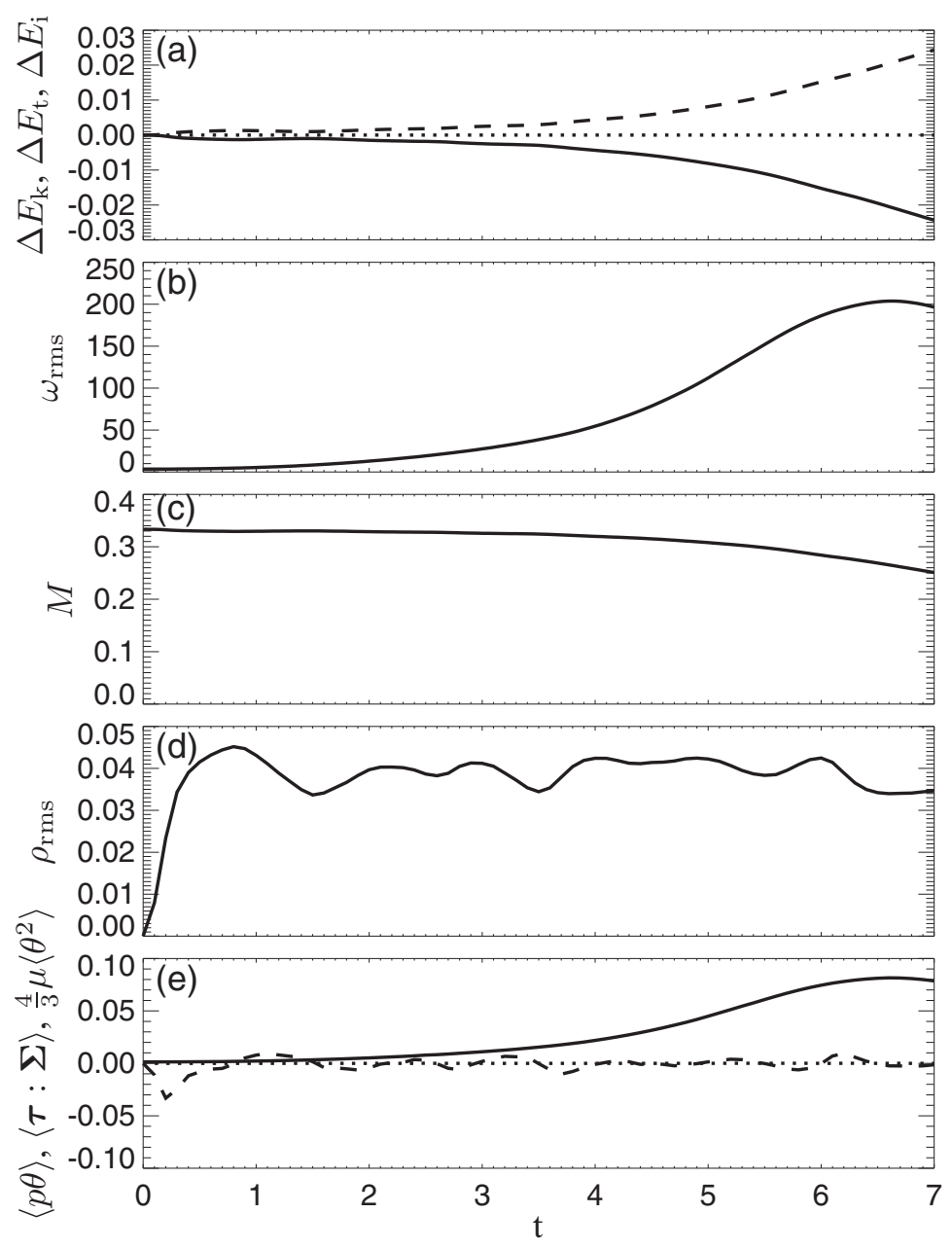

FIG. 1. Evolution in run 1: (a) the relative changes in the kinetic energy $\Delta E_{\mathrm{k}}$ (solid line), the total energy $\Delta E_{\mathrm{t}}$ (dotted line), and the internal energy $\Delta E_{\mathrm{i}}$ (dashed); (b) rms of the vorticity $\omega_{\text {rms }}$; (c) Mach number $M$; (d) rms of the density fluctuations $\rho_{\text {rms }}$; and (e) the dissipation rate $\langle\boldsymbol{\tau}: \boldsymbol{\Sigma}\rangle$ (solid line), the pressure-dilatation rate $\langle p \theta\rangle$ (dashed line), and the compressible dissipation rate $4 \mu\left\langle\theta^{2}\right\rangle / 3$ (dotted line) as functions of time.

The evolution of run 1 is shown in Fig. 1 . In this simulation, the total energy $E_{\mathrm{t}}=E_{\mathrm{k}}+E_{\mathrm{i}}$ is well conserved. Here $E_{\mathrm{k}}=\left\langle\rho u^{2}\right\rangle / 2$ is the kinetic energy and $E_{\mathrm{i}}=\langle\rho e\rangle$ is the internal one. Figure 1(a) displays the evolution of the relative changes in these energies, $\Delta E_{\mathrm{k}, \mathrm{i}, \mathrm{t}}=\left[E_{\mathrm{k}, \mathrm{i}, \mathrm{t}}(t)-E_{\mathrm{k}, \mathrm{i}, \mathrm{t}}(0)\right] / E_{\mathrm{t}}(0)$ (the solid line denotes the kinetic energy, the dashed line the internal one, and the dotted line denotes the total energy). The relative change of the total energy is negligible, $\left|\Delta E_{\mathrm{t}}(t=7)\right|<8 \times 10^{-6}$, and the kinetic energy is transformed to the internal one. Figure 1(b) shows the evolution of the rms of the vorticity $\boldsymbol{\omega}=\nabla \times \boldsymbol{u}, \omega_{\mathrm{rms}}^{2}=\left\langle|\boldsymbol{\omega}|^{2}\right\rangle$. The vorticity reaches the maximum of about 200 at $t=t_{\omega} \simeq 6.6$; this corresponds to the maximum of the (incompressible) dissipation rate, and it may be considered as a signature of a fully developed turbulent cascade in a decaying system. After about this time, the dissipation rate of the kinetic energy varies only slowly, indicating a quasistationary evolution [27]. Figure 1(c) displays the evolution of the average Mach number $M$ (i.e., the ratio between the rms of the velocity and the mean sound speed). $M$ slowly decreases during the evolution due to the decay of the level of fluctuations as well as due to the turbulent heating that leads to an 

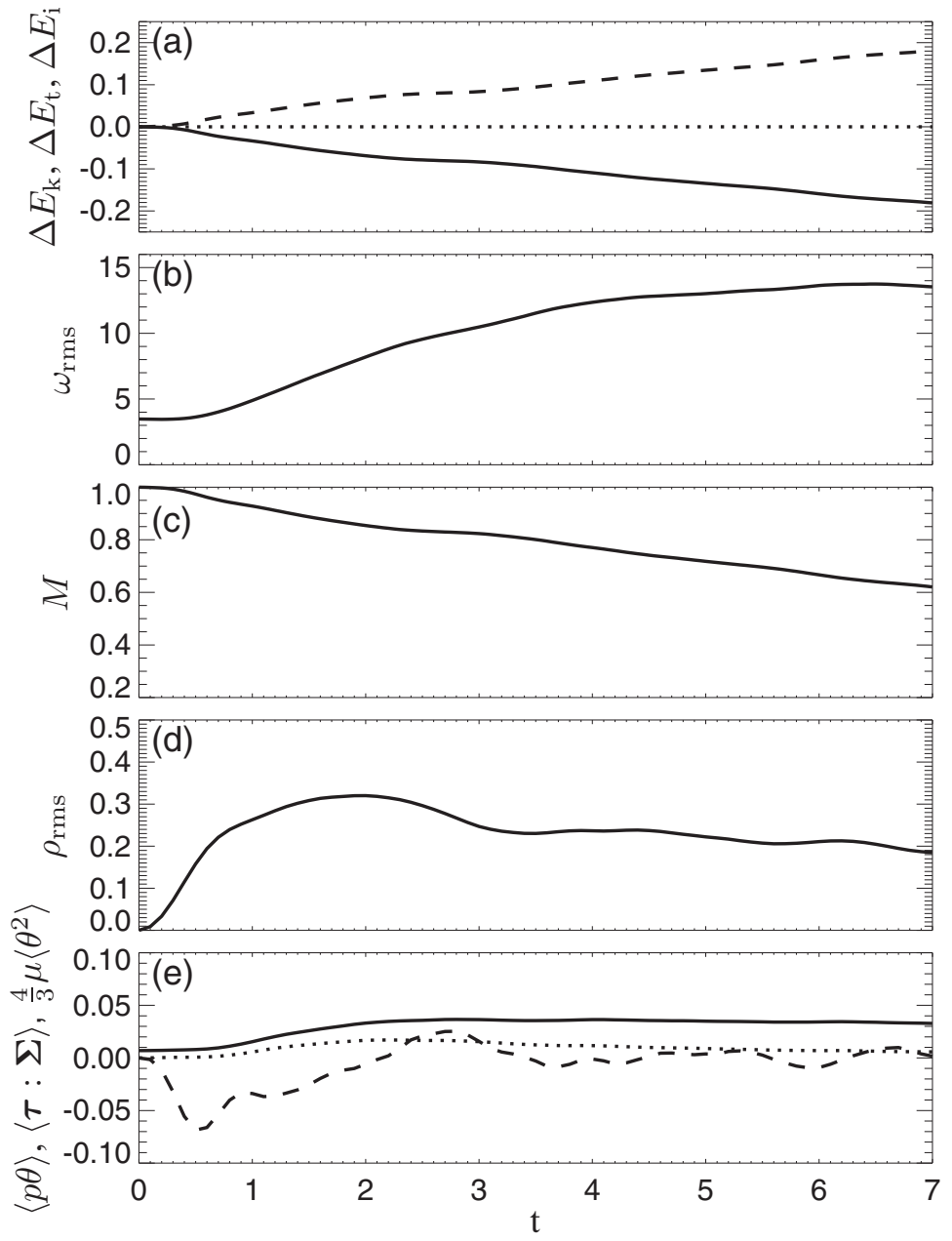

FIG. 2. Evolution in run 2: (a) the relative changes in the kinetic energy $\Delta E_{\mathrm{k}}$ (solid line), the total energy $\Delta E_{\mathrm{t}}$ (dotted line), and the internal energy $\Delta E_{\mathrm{i}}$ (dashed); (b) rms of the vorticity $\omega_{\mathrm{rms}}$; (c) Mach number $M$; (d) rms of the density fluctuations $\rho_{\text {rms }}$; and (e) the dissipation rate $\langle\boldsymbol{\tau}: \boldsymbol{\Sigma}\rangle$ (solid line), the pressure-dilatation rate $\langle p \theta\rangle$ (dashed line), and the compressible dissipation rate $4 \mu\left\langle\theta^{2}\right\rangle / 3$ (dotted line) as functions of time.

increasing sound speed. Figure 1(d) shows the rms of the density fluctuations, $\rho_{\text {rms }}^{2}=\left\langle\left(\rho-\rho_{0}\right)^{2}\right\rangle$ (where $\left.\rho_{0}=\langle\rho\rangle\right)$. Weak fluctuations $\left(\rho_{\text {rms }} \sim 0.04\right)$ develop during the first phase of the relaxation of the initial, constant $\rho$ conditions. Figure 1(e) quantifies the evolution of the dissipation rate $\langle\boldsymbol{\tau}: \boldsymbol{\Sigma}\rangle$ (solid line), the pressure dilatation term $\langle p \theta\rangle$ (dashed line), and the compressible dissipation term $4 \mu\left\langle\theta^{2}\right\rangle / 3$ (dotted line). In run 1 , the compressible dissipation is negligible, and the dissipation rate follows closely the behavior of the vorticity [see Fig. 1(b)]. A relatively large pressure-dilatation rate develops initially as a relaxation of the initial solenoidal conditions. At later times, the pressure dilatation becomes weaker than the dissipation rate and oscillates around zero [26,28]. Taking an average over about a period of these oscillations removes the exchange between the kinetic and internal energies induced by the pressure dilatation, $\langle\langle p \theta\rangle\rangle_{t} \simeq 0$; henceforth $\langle\bullet\rangle_{t}$ denotes time averaging.

Run 2 exhibits an evolution qualitatively similar to that of run 1 as shown in Fig. 2. Figure 2(a) displays the evolution of the relative changes in the kinetic, internal, and total energies; the relative 


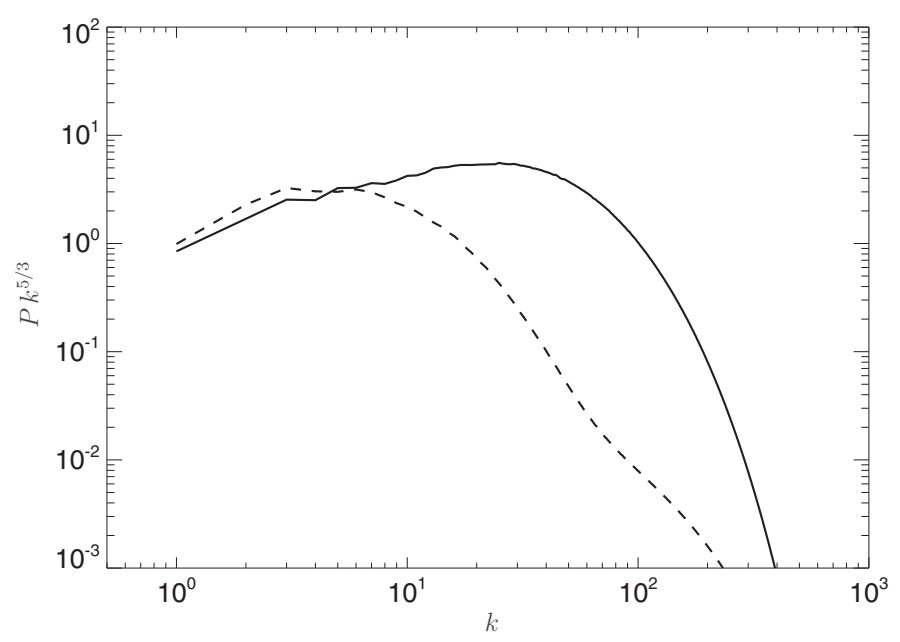

FIG. 3. Power spectral density of $\boldsymbol{u}$, compensated by $k^{5 / 3}$ as a function of the wave vector $k$ in run 1 (solid line) and run 2 (dashed line).

change of the total energy in run 2 is also negligible, $\left|\Delta E_{\mathrm{t}}(t=8)\right|<4 \times 10^{-5}$. Figure 2(b) shows that the rms of vorticity in run 2 reaches the maximum of about 14 at $t=t_{\omega} \simeq 6.5$. This is much smaller than that in run 1 likely due to the larger viscosity and compressibility. In run 2, the Mach number [Fig. 2(c)] decreases faster than in run 1 since the turbulent heating leads to larger relative changes of the temperature for the colder fluid. The larger Mach number leads to important density variations, and Fig. 2(d) shows that the rms of the density rapidly becomes about 0.3 . For later times, $\rho_{\text {rms }}$ tends to slowly decrease. Figure 2(e) shows the properties of dissipation and the pressure dilatation. In run 2, the compressible dissipation is not negligible, and, especially during the initial phase, compressible dissipation makes an important fraction of the total dissipation. The dissipation rate is interestingly smaller in run 2 compared to run 1 , whereas the pressure dilatation is more important. The kinetic energy decreases overall with time, whereas the internal energy increases due to the viscous dissipation. On top of this trend, both energies exhibit noticeable oscillations due to the pressure-dilatation-induced exchanges; these oscillations are also seen in the vorticity and the Mach number. As in run 1, the pressure dilatation at later times oscillates around zero and disappears when time-averaged.

Figure 3 shows the power spectral density (PSD) compensated by $k^{5 / 3}$ of the velocity fluctuation at the time $t_{\omega}, t_{\omega} \simeq 6.6$ for run 1 and $t_{\omega} \simeq 6.5$ for run 2 . Both PSDs exhibit hints of the Kolmogorovlike scaling; only a very small range of wave vectors have slopes compatible with $-5 / 3(k \in[12,30]$ and $k \in[3,6]$ for runs 1 and 2 , respectively) prior to the steepening due to the dissipation. The analysis of the energy transfer will show that only these scales can be roughly identified as the inertial range.

\section{SPECTRAL TRANSFER}

\section{A. Incompressible HD}

We start with the incompressible Navier-Stokes equation

$$
\frac{\partial \boldsymbol{u}}{\partial t}+(\boldsymbol{u} \cdot \nabla) \boldsymbol{u}=-\frac{\nabla p}{\rho}+v \Delta \boldsymbol{u},
$$

where $\boldsymbol{u}$ is the velocity field, $\rho$ is the density, $p$ is the pressure, and $v$ is the kinematic viscosity. In addition to the incompressibility, $\theta=0$, we also assume that the density is constant, $\rho=\rho_{0}$; 
henceforth, we set $\rho_{0}=1$. In this system, the equation for the kinetic energy (per mass) reads

$$
\frac{\partial}{\partial t}\left\langle\frac{1}{2}|\boldsymbol{u}|^{2}\right\rangle=-v\langle\nabla \boldsymbol{u}: \nabla \boldsymbol{u}\rangle=-\epsilon,
$$

where $\epsilon$ is the incompressible dissipation rate (per mass).

Taking the Fourier transform of Eq. (7), one gets an equation for the amplitude of a given Fourier mode,

$$
\begin{aligned}
& \widehat{\boldsymbol{u}}(\boldsymbol{k})=\sum_{\boldsymbol{x}} \boldsymbol{u}(\boldsymbol{x}) \exp (i \boldsymbol{k} \cdot \boldsymbol{x}), \\
& \frac{1}{2} \frac{\partial|\widehat{\boldsymbol{u}}|^{2}}{\partial t}+\operatorname{Re} \widehat{\boldsymbol{u}}^{*} \cdot(\widehat{\boldsymbol{u} \cdot \nabla}) \boldsymbol{u}=-v k^{2}|\widehat{\boldsymbol{u}}|^{2},
\end{aligned}
$$

where wide hats denote the Fourier transform, asterisks signify the complex conjugate, and $\mathrm{Re}$ means the real part.

For the kinetic energy in modes with wave-vector magnitudes smaller than or equal to $k$ (we take a low-pass filter in the Fourier space),

$$
E_{\mathrm{k} k}^{(i)}=\frac{1}{2} \sum_{\left|\boldsymbol{k}^{\prime}\right| \leqslant k}\left|\widehat{\boldsymbol{u}}\left(\boldsymbol{k}^{\prime}\right)\right|^{2},
$$

one gets this dynamic equation,

$$
\frac{\partial E_{\mathrm{k} k}^{(i)}}{\partial t}+S_{k}^{(i)}=-D_{k}^{(i)}
$$

where

$$
\begin{gathered}
S_{k}^{(i)}=\operatorname{Re} \sum_{\left|\boldsymbol{k}^{\prime}\right| \leqslant k} \widehat{\boldsymbol{u}}^{*}\left(\boldsymbol{k}^{\prime}\right) \cdot[\widehat{(\widehat{\boldsymbol{u} \cdot \nabla}) \boldsymbol{u}}]\left(\boldsymbol{k}^{\prime}\right), \\
D_{k}^{(i)}=v \sum_{\left|\boldsymbol{k}^{\prime}\right| \leqslant k}\left|\boldsymbol{k}^{\prime}\right|^{2}\left|\widehat{\boldsymbol{u}}\left(\boldsymbol{k}^{\prime}\right)\right|^{2} .
\end{gathered}
$$

Henceforth, the superscript ( $i$ ) denotes the incompressible approximation. In Eq. (11), $S_{k}^{(i)}$ describes the energy transfer (cascade) to scales with wave-vector magnitudes larger than $k$, whereas $D_{k}^{(i)}$ signifies the viscous dissipation on scales with wave-vector magnitudes smaller than or equal to $k$. Equation (11) may also serve to determine the inertial range as a region where

$$
S_{k}^{(i)}=\epsilon,
$$

i.e., where the energy transfer/cascade rate is equal to the dissipation one.

\section{B. Compressible HD}

To characterize the spectral decomposition of the kinetic energy in the compressible case, we define the density-weighted velocity field [29]

$$
\boldsymbol{w}=\rho^{1 / 2} \boldsymbol{u} .
$$

Taking the Fourier transform of Eq. (2), one gets an equation for an amplitude of a given Fourier mode $\widehat{\boldsymbol{w}}(\boldsymbol{k})$ as $[12,17]$

$$
\begin{aligned}
\frac{1}{2} \frac{\partial|\widehat{\boldsymbol{w}}|^{2}}{\partial t}= & -\operatorname{Re} \widehat{\boldsymbol{w}}^{*} \cdot\left(\widehat{\boldsymbol{u} \cdot \boldsymbol{\nabla}) \boldsymbol{w}}-\frac{1}{2} \operatorname{Re} \widehat{\boldsymbol{w}}^{*} \cdot \widehat{\theta \boldsymbol{w}}\right. \\
& -\widehat{\boldsymbol{w}}^{*} \cdot \widehat{\rho^{-1 / 2} \nabla} p+\widehat{\boldsymbol{w}}^{*} \cdot \rho^{-\widehat{-1 / 2} \nabla} \cdot \boldsymbol{\tau}
\end{aligned}
$$


For the kinetic energy in modes with wave-vector magnitudes smaller than or equal to $k$,

$$
E_{\mathrm{k} k}=\frac{1}{2} \sum_{\left|\boldsymbol{k}^{\prime}\right| \leqslant k}\left|\widehat{\boldsymbol{w}}\left(\boldsymbol{k}^{\prime}\right)\right|^{2},
$$

one gets, analogously to the incompressible case, the following equation:

$$
\frac{\partial E_{\mathrm{k} k}}{\partial t}+S_{k}=\Psi_{k}-D_{k}
$$

where (henceforth we will drop the $\boldsymbol{k}^{\prime}$ argument)

$$
\begin{gathered}
S_{k}=\operatorname{Re} \sum_{\left|\boldsymbol{k}^{\prime}\right| \leqslant k} \widehat{\boldsymbol{w}}^{*} \cdot \widehat{(\boldsymbol{u} \cdot \boldsymbol{\nabla}) \boldsymbol{w}}+\frac{1}{2} \operatorname{Re} \sum_{\left|\boldsymbol{k}^{\prime}\right| \leqslant k} \widehat{\boldsymbol{w}}^{*} \cdot \widehat{\theta \boldsymbol{w}}, \\
\Psi_{k}=-\operatorname{Re} \sum_{\left|\boldsymbol{k}^{\prime}\right| \leqslant k} \widehat{\boldsymbol{w}}^{*} \cdot \rho^{-1 / 2} \boldsymbol{\nabla}, \\
D_{k}=-\operatorname{Re} \sum_{\left|\boldsymbol{k}^{\prime}\right| \leqslant k} \widehat{\boldsymbol{w}}^{*} \cdot \rho^{-\widehat{-1 / 2} \boldsymbol{\nabla}} \cdot \boldsymbol{\tau} .
\end{gathered}
$$

Here $S_{k}$ represents the energy transfer/cascade rate, $\Psi_{k}$ describes the pressure-dilatation effect, and $D_{k}$ is the dissipation rate for modes with wave-vector magnitude smaller than or equal to $k$. For large wave vectors, one gets unfiltered values,

$$
E_{\mathrm{k} k} \rightarrow E_{\mathrm{k}}, \quad \Psi_{k} \rightarrow\langle p \theta\rangle, \quad \text { and } \quad D_{k} \rightarrow Q_{\mu},
$$

where $Q_{\mu}$ is the viscous dissipation rate, $Q_{\mu}=\langle\boldsymbol{\tau}: \boldsymbol{\Sigma}\rangle$. The inertial range could be defined as

$$
S_{k}=Q_{\mu}
$$

but this equation neglects the pressure dilatation.

To validate the conservation of energy at any given scale, expressed by Eqs. (18) and (11), and to compare the incompressible and compressible decomposition, we introduce the error, i.e., the departure from the conservation of energy, for the compressible case

$$
O_{k}=-\frac{\partial E_{\mathrm{k} k}}{\partial t}-S_{k}+\Psi_{k}-D_{k}
$$

and for the incompressible case

$$
O_{k}^{(i)}=-\frac{\partial E_{\mathrm{k} k}^{(i)}}{\partial t}-S_{k}^{(i)}-D_{k}^{(i)}
$$

Figure 4(a) displays the results of the spectral transfer analysis for run 1; solid lines show the following: black, $O_{k}$ and its contributions; blue, the rate of change/decaying term $-\partial E_{\mathrm{k} k} / \partial t$; green, the energy transfer/cascade term $-S_{k}$; orange, the pressure dilatation term $\Psi_{k}$; and red, the dissipation term $-D_{k}$ (all normalized with respect to $Q_{\mu}$ ) as functions of $k$. Dashed lines show the corresponding error of the incompressible approximation $O_{k}^{(i)}$ and its contributions. The validity tests $O_{k}$ and $O_{k}^{(i)}$ in Fig. 4(a) are calculated at $t_{\omega}$ and $t_{\omega}+\Delta t$ with $\Delta t=0.1$, and $\partial E_{\mathrm{k} k} / \partial t$ is approximated by the finite difference $\partial E_{\mathrm{k} k} / \partial t \approx\left[E_{\mathrm{k} k}\left(t_{\omega}+\Delta t\right)-E_{\mathrm{k} k}\left(t_{\omega}\right)\right] / \Delta t$. Equation (18) is well satisfied, $\left|O_{k}\right| / Q_{\mu}<0.01$; the error is partly numerical, likely related to the finite-difference approximation of $\partial E_{\mathrm{k} k} / \partial t$. The rate of change of the kinetic energy $\partial E_{\mathrm{k} k} / \partial t$ is negative and varies mostly on large scales; the energy-containing range is then on large scales, roughly for wave-vector magnitudes smaller than about 3 . The spectral energy transfer/cascade rate $S_{k}$ dominates on medium scales, with a maximum around $k=20$. The viscous dissipation $D_{k}$ is important on small scales. The pressure dilatation is weak in this weakly compressible case; the incompressible predictions are close to their compressible counterparts. The error of the incompressible approach $O_{k}^{(i)}$ appears to be related to the neglected pressure-dilatation term. 

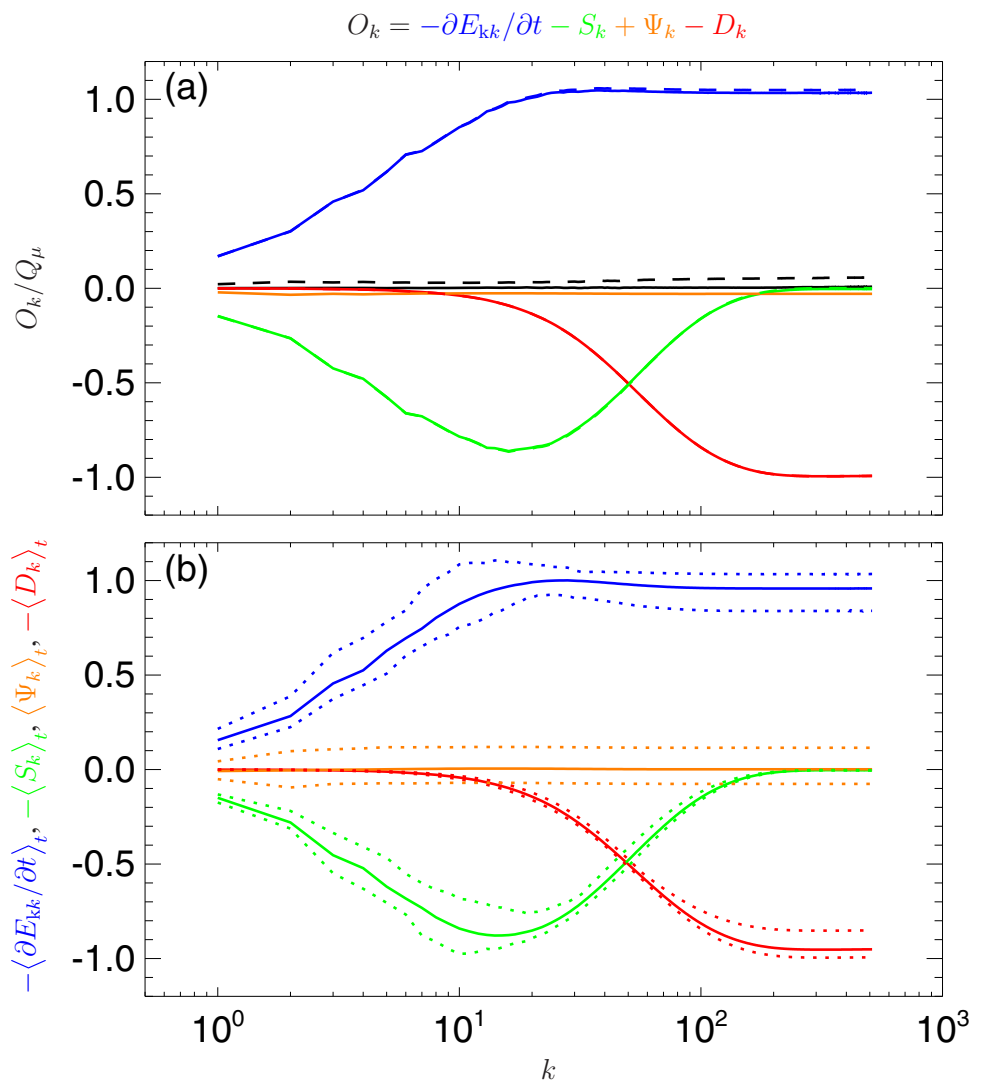

FIG. 4. Spectral transfer in run 1. (a) The validity test $O_{k}$ of Eq. (24) (black solid line) as a function of $k$ along with the different contributions (solid lines): blue, the losses/decay term $-\partial E_{\mathrm{k} k} / \partial t$; green, the transfer term $-S_{k}$; orange, the pressure dilatation term $\Psi_{k}$; and red, the dissipation term $-D_{k}$. Dashed lines show the incompressible equivalent $O_{k}^{(i)}$ [Eq. (25)] and its contributions. (b) Time-averaged contributing terms (solid lines) with their minimum and maximum values (dotted lines) for blue, the decay $-\left\langle\partial E_{\mathrm{k} k} / \partial t\right\rangle_{t}$; green, the transfer $-\left\langle S_{k}\right\rangle_{t}$; orange, the pressure-dilatation term $\left\langle\Psi_{k}\right\rangle_{t}$; and red, the dissipation term $-\left\langle D_{k}\right\rangle_{t}$. All the quantities are normalized with respect to $Q_{\mu}$.

In run 1, the pressure-dilatation effect is small but non-negligible at a given time. As the pressure dilatation oscillates in time, it is interesting to look at the time-averaged quantities in Eq. (18). Figure 4(b) displays the different terms averaged over the time 5.8-7.0 (during this period, the system is quasistationary; see Fig. 1) by solid lines. The dotted lines show the corresponding maximum and minimum values. There we see that even in the weakly compressible run 1 , the different terms fluctuate with an important amplitude (of the order of $0.1 Q_{\mu}$ ). However, the pressure dilatation is, on average, negligible on all scales. Finally, we note that in run 1 there is no inertial range as $S_{k}$ reaches maximally about $0.8 Q_{\mu}$ in a region where the dissipation is not negligible.

Results from the ST approach in run 2 are shown in Fig. 5 in the same format as in Fig. 4. Figure 5(a) is obtained for the times $t_{\omega}$ and $t_{\omega}+\Delta t$ as above. Equation (18) is well satisfied in run 2, $\left|O_{k}\right| / Q_{\mu}<0.005$. Figure 5(a) shows that the region dominated by dissipation is wider compared to run 2 due to the larger viscosity. The energy-containing region as well as the region where the energy transfer dominates are shifted to larger scales. Figure 5(a) demonstrates the cumulative behavior of the low-pass filter in $k$ space, Eq. (22). The pressure-dilatation term is stronger compared to that in run 1 and reaches the largest value on large $k$ (small scales). The error of the incompressible 


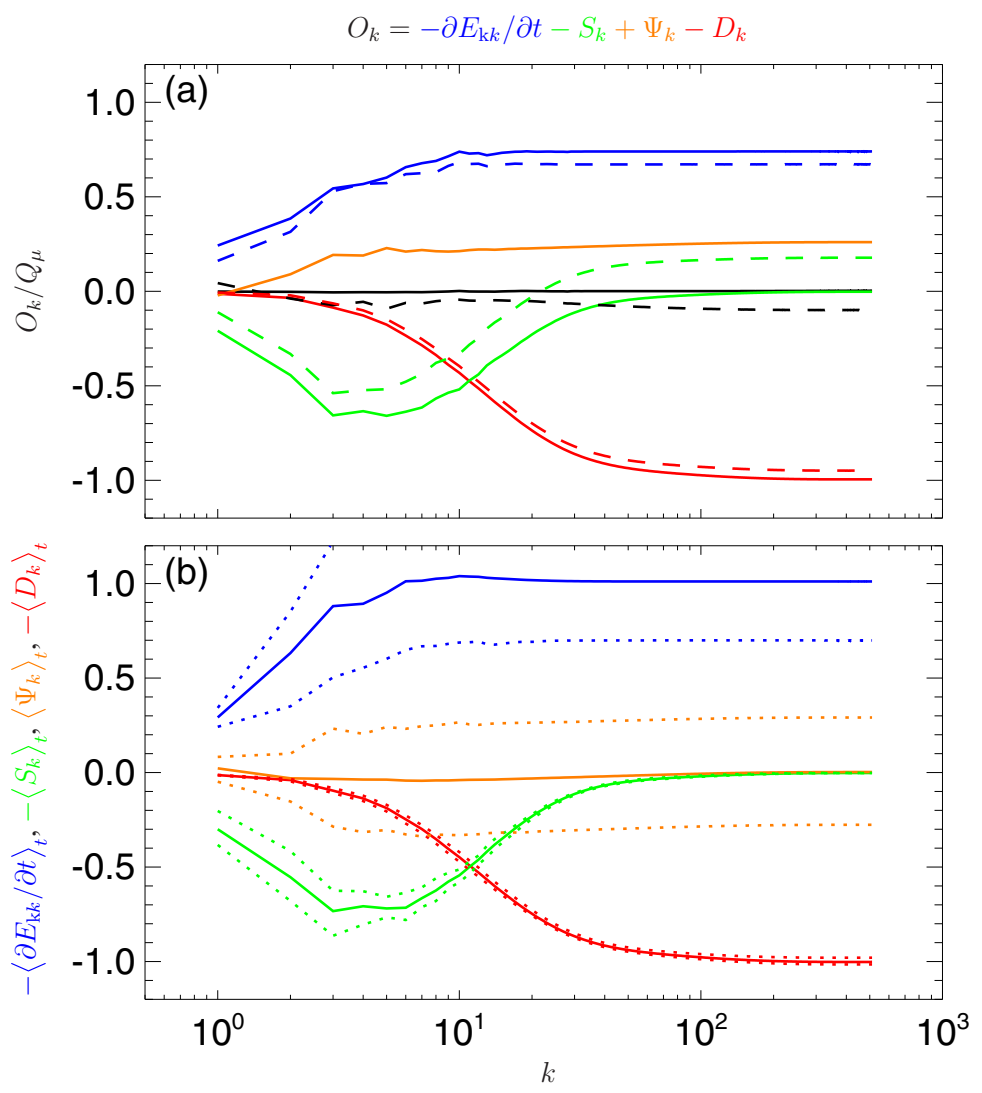

FIG. 5. Spectral transfer in run 2 in the same format as in Fig. 4.

approach $O_{k}^{(i)}$ is larger and is not only connected with the pressure dilatation; the incompressible terms (especially the cascade one) differ noticeably from the compressible ones.

Figure 5(b) shows that over the pressure-dilatation period, the different components, $-\partial E_{\mathrm{k} k} / \partial t$, $-S_{k}, \Psi_{k}$, and $-D_{k}$, have very large temporal variations [large differences between the minimum and maximum values given by the dotted lines in Fig. 5(b)]. The averaged pressure-dilatation term is weak and exhibits small negative values over medium scales, a behavior qualitatively similar to that of the transfer $-S_{k}$. This indicates that the averaged effect of the pressure dilatation is a spectral transfer of the kinetic energy to smaller scales without a net exchange between kinetic and internal energies.

The observed spectra in Fig. 3 can now be interpreted using Figs. 4 and 5. The regions where the compensated power spectra $P k^{5 / 3}$ are about flat correspond to regions where the energy transfer rate $S_{k}$ dominates. Large scales are dominated by the decay of $E_{\mathrm{k} k}$ and smaller scales are dominated by the dissipation.

\section{KÁRMÁN-HOWARTH-MONIN EQUATION}

\section{A. Incompressible HD}

In the incompressible HD [see Eq. (7)], the structure function

$$
\mathcal{S}^{(i)}(\boldsymbol{l})=\left\langle|\delta \boldsymbol{u}|^{2}\right\rangle
$$


[where $\delta \boldsymbol{u}=\boldsymbol{u}\left(\boldsymbol{x}^{\prime}\right)-\boldsymbol{u}(\boldsymbol{x}), \boldsymbol{x}^{\prime}=\boldsymbol{x}+\boldsymbol{l}$, and $\langle\bullet\rangle$ denotes spatial averaging] describes the kineticenergy (per mass) spatial scale distribution and is related to the kinetic-energy power spectrum [1]. For statistically homogeneous decaying turbulence, one can get the following dynamic KHM equation for the $\mathcal{S}^{(i)}[6,7]$ :

$$
\frac{\partial \mathcal{S}^{(i)}}{\partial t}+\nabla_{l} \cdot \mathcal{Y}^{(i)}=2 v \Delta_{l} \mathcal{S}^{(i)}-4 \epsilon
$$

where

$$
\mathcal{Y}^{(i)}(\boldsymbol{l})=\left\langle\delta \boldsymbol{u}|\delta \boldsymbol{u}|^{2}\right\rangle
$$

$\epsilon$ is the incompressible heating rate [see Eq. (8)]. Equation (27) is simply related to its original form, which involves the cross-correlation $R(\boldsymbol{l})=\left\langle\boldsymbol{u}\left(\boldsymbol{x}^{\prime}\right) \cdot \boldsymbol{u}(\boldsymbol{x})\right\rangle[1]$,

$$
2 \frac{\partial R(\boldsymbol{l})}{\partial t}-\nabla_{\boldsymbol{l}} \cdot \mathcal{Y}^{(i)}=4 v \Delta_{l} R(\boldsymbol{l})
$$

since $S^{(i)}=2\left\langle|\boldsymbol{u}|^{2}\right\rangle-2 R(\boldsymbol{l})$ and $\partial\left\langle|\boldsymbol{u}|^{2}\right\rangle / \partial t=-2 \epsilon$. Equation (27) relates the change of the secondorder structure function $\mathcal{S}^{(i)}, \partial \mathcal{S}^{(i)} / \partial t$, the dissipation rate $\epsilon$, the cross-scale transfer/cascade rate $\mathcal{K}^{(i)}=-\nabla_{\boldsymbol{l}} \cdot \mathcal{Y}^{(i)} / 4$, and the dissipation term $v \Delta_{l} \mathcal{S}^{(i)}$ (henceforth we drop the $\boldsymbol{l}$ subscript for $\boldsymbol{\nabla}$ and $\Delta$ ). The inertial range can be formally defined as the region where the decay and dissipation terms are negligible, so that

$$
\mathcal{K}^{(i)}=\epsilon .
$$

For isotropic media, in the infinite Reynolds number limit, Eq. (30) leads to the so called exact (scaling) laws [1,8]. Equation (27) is more general and may be directly tested in numerical simulations [30], since large Reynolds numbers needed for existence of the inertial range are computationally challenging [31].

\section{B. Compressible HD}

For the compressible Navier-Stokes equations (1) and (2), one possibility to describe the scale distribution of kinetic energy is the structure function $\mathcal{S}=\langle\delta \boldsymbol{u} \cdot \delta(\rho \boldsymbol{u})\rangle$ [21]. For the statistically homogeneous system, one gets

$$
\frac{\partial \mathcal{S}}{\partial t}+\nabla \cdot \mathcal{Y}+\mathcal{R}=4 \Psi-4 \mathcal{D}+C_{\Psi}-C_{D},
$$

where $\mathcal{Y}=\langle\delta \boldsymbol{u}[\delta(\rho \boldsymbol{u}) \cdot \delta \boldsymbol{u}]\rangle, \mathcal{R}=\left\langle\delta \boldsymbol{u} \cdot\left(\theta^{\prime} \rho \boldsymbol{u}-\theta \rho^{\prime} \boldsymbol{u}^{\prime}\right)\right\rangle, \Psi=\langle\delta p \delta \theta\rangle / 2$, and $\mathcal{D}=\langle\delta \boldsymbol{\tau}: \delta \boldsymbol{\Sigma}\rangle / 2$.

Here $C_{\Psi}$ and $C_{\mathcal{D}}$ are correction terms to $\Psi$ and $\mathcal{D}$ (that we choose to represent the pressure dilatation and the dissipation), respectively,

$$
C_{\Psi}=\mathcal{C}_{\rho}[\boldsymbol{u}, \nabla p], \quad C_{\mathcal{D}}=\mathcal{C}_{\rho}[\boldsymbol{u}, \nabla \cdot \tau],
$$

where

$$
\mathcal{C}_{\rho}[\boldsymbol{a}, \boldsymbol{b}]=\left(\frac{\rho^{\prime}}{\rho}-1\right) \boldsymbol{a}^{\prime} \cdot \boldsymbol{b}+\left(\frac{\rho}{\rho^{\prime}}-1\right) \boldsymbol{a} \cdot \boldsymbol{b}^{\prime} .
$$

Note that the $C_{\Psi}$ and $C_{\mathcal{D}}$ terms depend explicitly on the level of density fluctuations in the system.

$\mathcal{S}$ and $\mathcal{Y}$ are compressible generalizations of $\mathcal{S}^{(i)}$ and $\mathcal{Y}^{(i)}$, respectively. The $\mathcal{R}$ term presents an additional compressible energy-transfer channel [21] and likely corresponds to the compressible part in the spectral transfer, Eq. (19); we do not see an obvious way how to turn this term to a divergence form similar to $\nabla \cdot \mathcal{Y}$. The term $\Psi$ is a structure-function formulation of the pressure dilatation effect $p \theta$. The viscous term $\mathcal{D}$ corresponds to a combination of the two dissipation terms in the incompressible case, $\epsilon-v \Delta \mathcal{S}^{(i)} / 2$, in Eq. (27). On large scales, $|\delta \boldsymbol{x}| \rightarrow \infty$, the correlations 


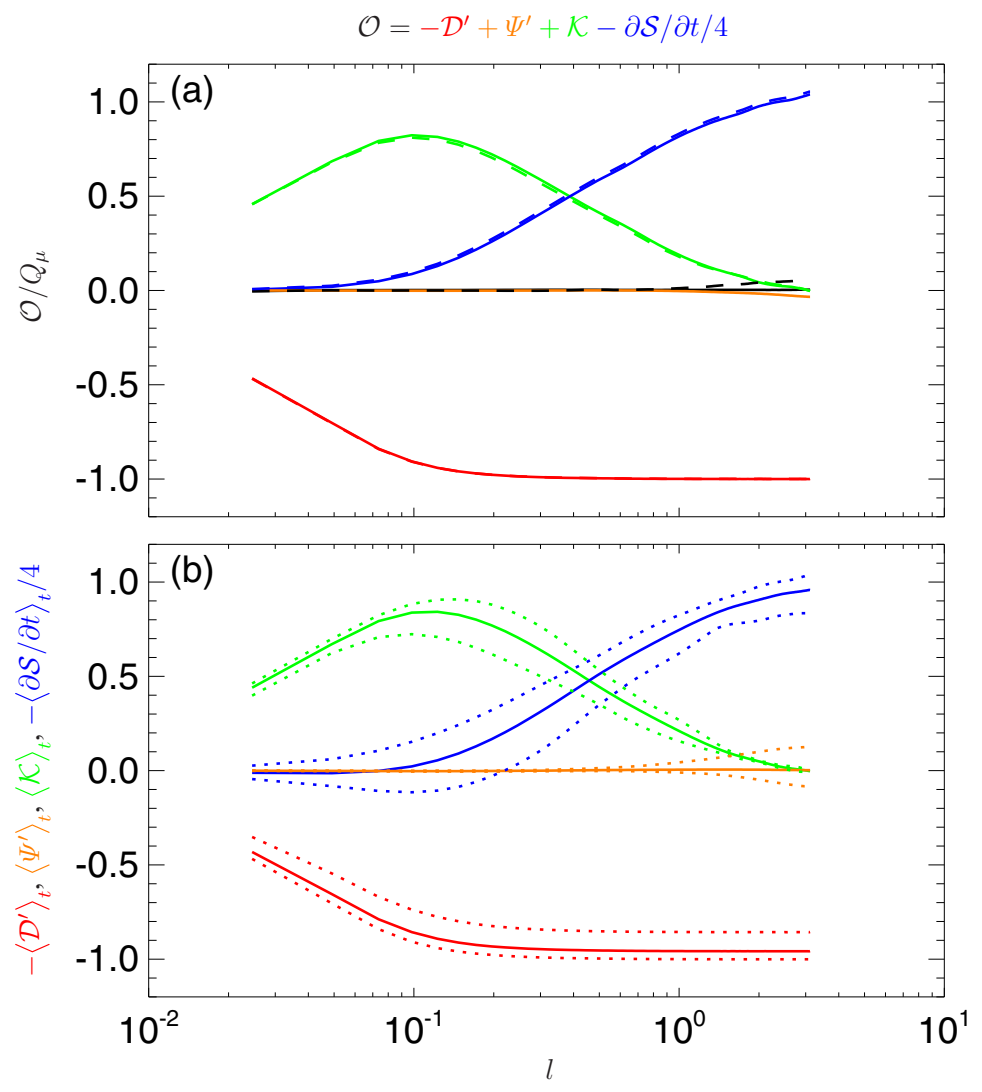

FIG. 6. KHM equation for run 1: (a) The validity test $O$ of Eq. (34) (black solid line) as a function of the separation scale $l$ along with the different contributions: blue, $-\partial \mathcal{S} / \partial t / 4$; green, $\mathcal{K}$; orange, $\Psi^{\prime}$; and red, $-D^{\prime}$. Dashed lines show the incompressible equivalents. (b) Time-averaged contributing terms (solid lines) with their minimum and maximum values (dotted lines) for blue, $-\langle\partial \mathcal{S} / \partial t\rangle_{t} / 4$; green, $\langle\mathcal{K}\rangle_{t} / 4$; orange, $\left\langle\Psi^{\prime}\right\rangle_{t}$; and red, $-\left\langle\mathcal{D}^{\prime}\right\rangle_{t}$. All the quantities are normalized with respect to $Q_{\mu}$.

$\left\langle\boldsymbol{\tau}\left(\boldsymbol{x}^{\prime}\right): \boldsymbol{\Sigma}\right\rangle \rightarrow 0$, and the viscous term becomes the viscous heating rate $Q_{\mu}$,

$$
\mathcal{D} \rightarrow\langle\tau: \Sigma\rangle=Q_{\mu} .
$$

The inertial range may be defined as the interval in the space of separation scales $l$ where

$$
\mathcal{K}=Q_{\mu},
$$

where $\mathcal{K}=-(\nabla \cdot \mathcal{Y}+\mathcal{R}) / 4$ is the cascade/energy transfer term. Equation (33) corresponds to the ST relation [Eq. (23)] and also neglects the pressure-dilatation effect. Now we can use Eq. (31) to interpret the simulation results. We define the departure from zero of this equation as

$$
O(l)=-\frac{1}{4} \frac{\partial \mathcal{S}}{\partial t}+\mathcal{K}+\Psi^{\prime}-\mathcal{D}^{\prime}
$$

where the correction terms were included in $\Psi^{\prime}$ and $\mathcal{D}^{\prime}, \Psi^{\prime}=\Psi+C_{\Psi} / 2$ and $\mathcal{D}^{\prime}=\mathcal{D}+C_{\mathcal{D}} / 2$.

The calculation of structure functions in $3 \mathrm{D}$ is computationally demanding, thus the KHM analysis is done on a $256^{3}$ box (taking every fourth point in all directions). The structure functions are calculated over the $3 \mathrm{D}$ separation space and isotropized/averaged over the solid angle. The partial time derivative is approximated by the finite difference between the two times. Figure 6(a) 
shows the validity test $O$ in run 1 as a function of the scale $l=|\boldsymbol{l}|$ along with the different contributions, the decay term $-\partial \mathcal{S} / \partial t / 4$, the energy transfer/cascade term $\mathcal{K}$, the pressure dilatation term $\Psi^{\prime}$, and the dissipation term $-\mathcal{D}^{\prime}$. Equation (31) is well satisfied in run 1, and the departure from validity is small, $|O| / Q_{\mu}<0.005$; this error is due to the finite-difference estimation of $\partial \mathcal{S} / \partial t$ (as in the spectral transfer case).

On large scales, the compressible dissipation term $\mathcal{D} \rightarrow Q_{\mu}$ as expected. Similarly, $\partial \mathcal{S} / \partial t / 4 \rightarrow$ $\partial E_{\mathrm{k}} / \partial t \sim-Q_{\mu}$. The pressure-dilatation term is small and appears only on large scales. The cascade term is important on medium scales, but there is no true inertial range, since both the decay and the dissipation are not negligible there. Run 1 is weakly compressible; the compressible energy-transfer term $\mathcal{R}$ is small $\left(|\mathcal{R}| / Q_{\mu}<0.03\right)$. Also, the correction terms are negligible $\left(\left|C_{\Psi}\right| / Q_{\mu}<0.006\right.$ and $\left.\left|C_{\mathcal{D}}\right| / Q_{\mu}<0.002\right)$.

Figure 6(a), using dashed lines, displays the results of the corresponding incompressible version of the KHM equation, with the validity test given by

$$
O^{(i)}(l)=-\frac{1}{4} \frac{\partial \mathcal{S}^{(i)}}{\partial t}+\mathcal{K}^{(i)}+\frac{1}{2} \nu \Delta \mathcal{S}^{(i)}-\epsilon .
$$

The incompressible terms are comparable to their compressible counterparts; in particular, the dissipation terms are close to each other since the dissipation is mostly incompressible [see Fig. 1(e)]. The incompressible error $O^{(i)}$ appears on large scales and is related to the missing pressure-dilatation term, in agreement with the ST results.

Figure 6(b) displays the results of the KHM equation averaged over one pressure-dilatation oscillation period. The colored solid lines show the time-averaged quantities, the decay term $-\partial \mathcal{S} / \partial t / 4$, the energy transfer/cascade term $\mathcal{K}$, the pressure dilatation term $\Psi^{\prime}$, and the scaledependent dissipation term $-\mathcal{D}^{\prime}$. The colored dotted lines show the corresponding minimum and maximum values. The averaged pressure-dilatation effect is negligible even though the variation is of the order $0.1 Q_{\mu}$. Similar variations are seen also in other terms.

The results for the more compressible run 2 are shown in Fig. 7. Figure 7(a), using solid lines, displays the results for the time $t_{\omega}$ and $t_{\omega}+\Delta t$, with the error check $O$ as a function of $l$ along with the different contributions. Equation (31) is also well satisfied in run 2, and the departure from validity is small, $|O| / Q_{\mu}<0.01$. The KHM approach exhibits cumulative properties similar to those of the ST approach [Eq. (22)]. The compressible dissipation term $\mathcal{D} \rightarrow Q_{\mu}$ on large scales as expected, Similarly, $\partial \mathcal{S} / \partial t / 4 \rightarrow \partial E_{\mathrm{k}} / \partial t \simeq-0.7 Q_{\mu}$ and $\Psi^{\prime} \rightarrow\langle p \theta\rangle \simeq 0.3 Q_{\mu}$. On large scales, we recover the energy conservation $\partial E_{\mathrm{k}} / \partial t=\langle p \theta\rangle-Q_{\mu}$.

Dashed lines in Fig. 7(a) show the incompressible results, $O^{(i)}$, and its constituents. The incompressible KHM equation is not applicable in run 2: the error $O^{(i)}$ is substantial and is not simply related to the pressure dilatation; an important part of dissipation is compressible [see Fig. 2(e)], and the incompressible transfer rate, $\mathcal{K}^{(i)}$, departs strongly from the compressible one, $\mathcal{K}$. This is partly due to the compressible energy-transfer term $\mathcal{R}$ that becomes important $\left(|\mathcal{R}| / Q_{\mu}<0.7\right)$. In run 2, the correction terms are not negligible $\left(\left|C_{\Psi}\right| / Q_{\mu}<0.3\right.$ and $\left.\left|C_{\mathcal{D}}\right| / Q_{\mu}<0.1\right)$.

It is interesting that for run 2 the incompressible approximation overestimates the energy-transfer rate in the KHM approach, whereas for the ST method the incompressible equation gives an energy-transfer rate that is lower than the compressible one [see Fig. 5(a)]. The incompressible approximation is not generally valid; however, it may possibly be useful to locate the inertial range.

The different contributing terms of Eq. (31) time-averaged over one pressure-dilatation oscillation are shown in Fig. 7(b). All the quantities (except the dissipation one) exhibit large variations, mainly on large scales. The averaged pressure-dilatation term, $\left\langle\Psi^{\prime}\right\rangle_{t}$, is about zero on large and small scales and reaches the maximum $\simeq 0.05 Q_{\mu}$ at about $l=20$. The positive value of $\left\langle\Psi^{\prime}\right\rangle_{t}$ suggests that the pressure-dilatation effect leads to a transfer of the kinetic energy from large to smaller scales, while there is no net energy exchange between the kinetic and internal energies, in agreement with the ST results.

Note that the choice $\delta(\rho \boldsymbol{u}) \cdot \delta \boldsymbol{u}$ corresponds in the ST approach to $\operatorname{Re} \widehat{\rho \boldsymbol{u}} \cdot \widehat{\boldsymbol{u}}^{*}$ [32]. For the ST equation with $|\widehat{\boldsymbol{w}}|^{2}[12,17]$ [see Eq. (18)] one can obtain an alternative KHM equation taking 

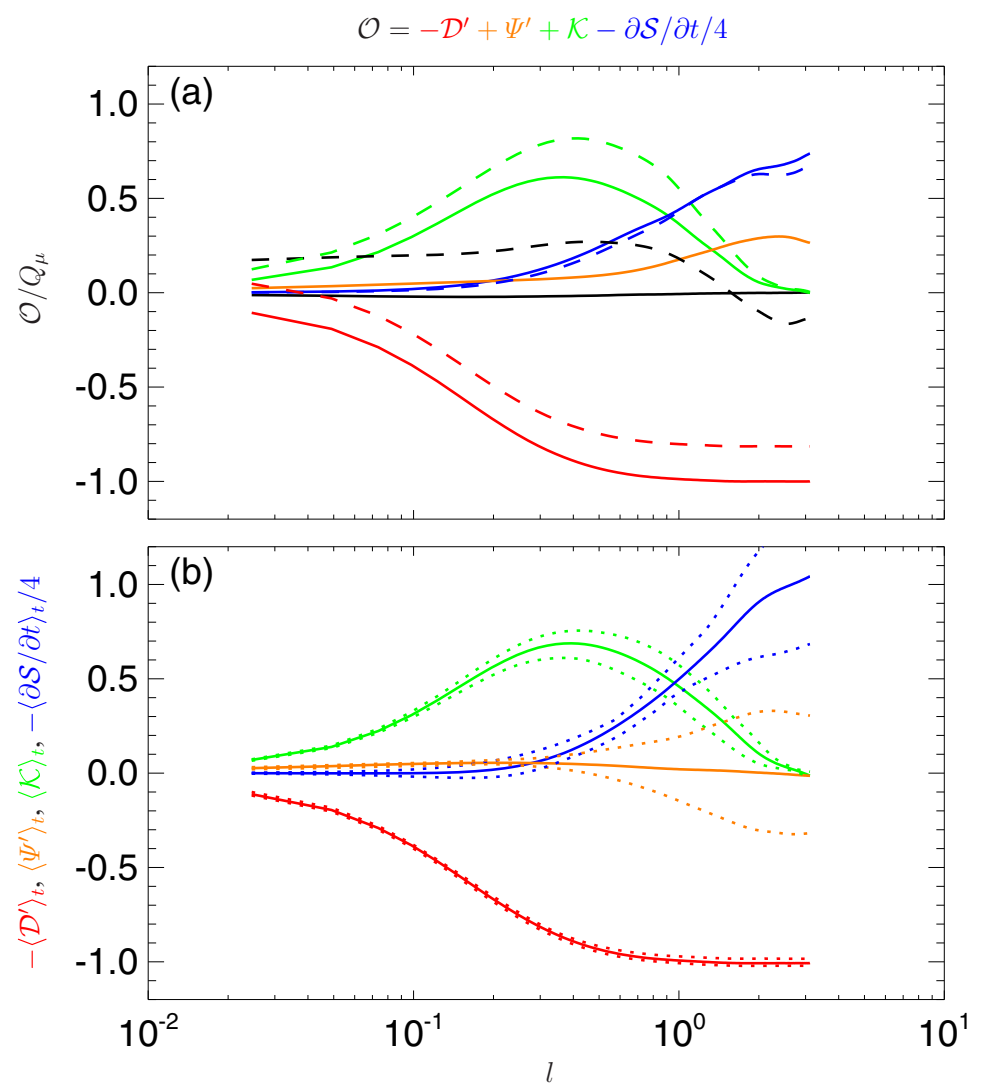

FIG. 7. KHM equation for run 2 in the same format as in Fig. 6.

$\mathcal{S}_{w}=\left\langle|\delta \boldsymbol{w}|^{2}\right\rangle$ as

$$
\frac{\partial \mathcal{S}_{w}}{\partial t}+\nabla_{l} \cdot \mathcal{Y}_{w}+\mathcal{R}_{w}=4 \Psi-4 \mathcal{D}+C_{\Psi w}-C_{\mathcal{D} w}
$$

where

$$
\begin{aligned}
& \mathcal{Y}_{w}=\left\langle\delta \boldsymbol{u}|\delta \boldsymbol{w}|^{2}\right\rangle, \quad \mathcal{R}_{w}=\left\langle\delta \boldsymbol{w} \cdot\left(\theta^{\prime} \boldsymbol{w}-\theta \boldsymbol{w}^{\prime}\right)\right\rangle, \\
& C_{\Psi w}=2 \mathcal{C}_{\sqrt{\rho}}[\boldsymbol{u}, \nabla p], \quad C_{\mathcal{D} w}=2 \mathcal{C}_{\sqrt{\rho}}[\boldsymbol{u}, \nabla \cdot \boldsymbol{\tau}] .
\end{aligned}
$$

For the two weakly compressible runs presented here, these two variants of the KHM relation give almost identical results.

\section{Comparison}

For both the runs, the ST and KHM equations give quantitatively analogous results. This is not surprising. $E_{\mathrm{k} k}$ represents a low-pass filtered spectral distribution of the kinetic energy, whereas $\mathcal{S}$ represents the kinetic energy at the separation scales smaller than $l$ (corresponding to a high-pass filter), and similar differences apply to the other terms. A remaining question is the relationship between the wave vector $k$ and the scale separation $l$. Since the two quantities should be inversely proportional, we tested different factors $\alpha$ in $k=\alpha / l$. For $\alpha=\sqrt{3}$ the ST and KHM results get close to each other. Figure 8 shows that in both runs, for $k=\sqrt{3} / l$, the time-averaged cascade rates 

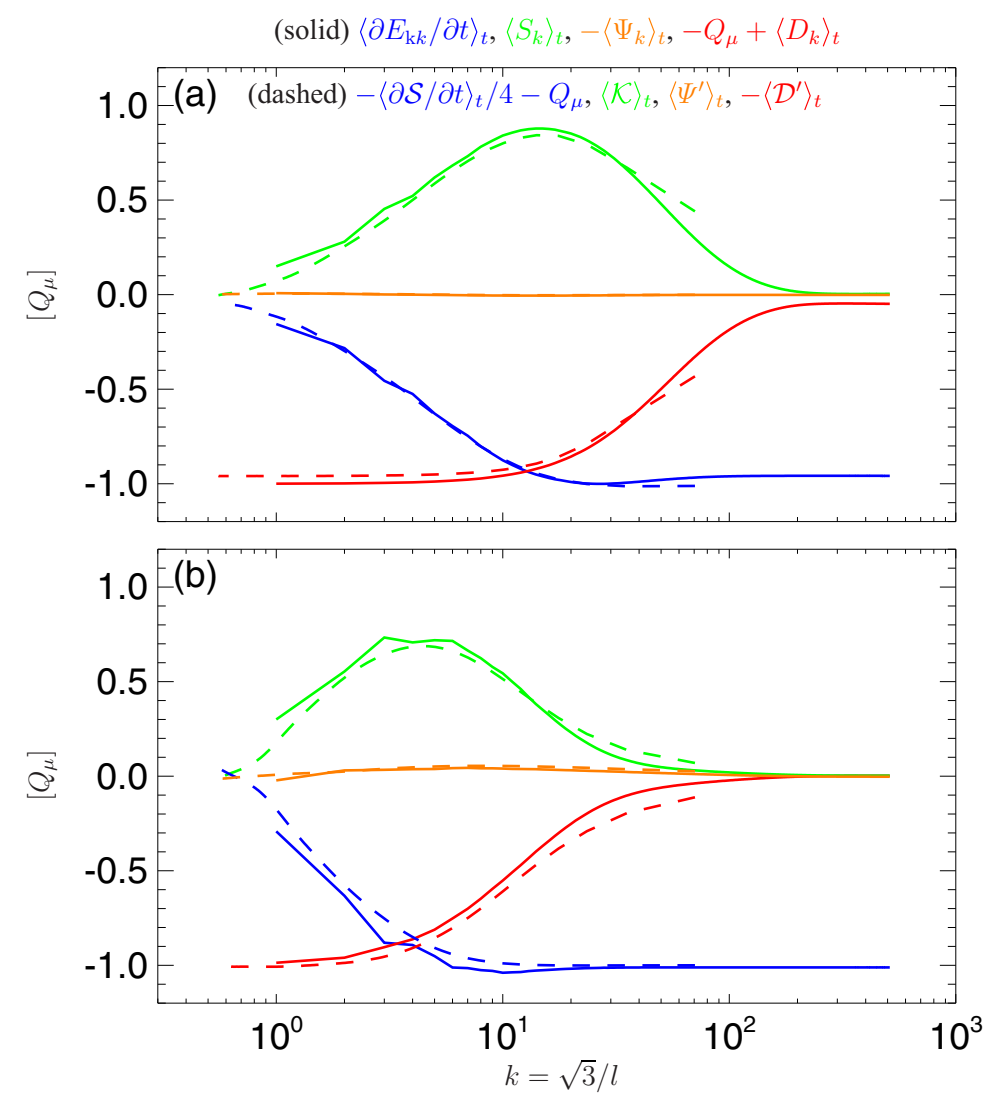

FIG. 8. Direct comparison between ST and KHM methods for (a) run 1 and (b) run 2: Solid lines denote the time-averaged ST terms (see Figs. 4 and 5): blue, the losses/decay $\left\langle\partial E_{\mathrm{k} k} / \partial t\right\rangle_{t}$; green, the transfer $\left\langle S_{k}\right\rangle_{t}$; orange, the pressure-dilatation term $-\left\langle\Psi_{k}\right\rangle_{t}$; and red, the dissipation term $\left\langle D_{k}\right\rangle_{t}-Q_{\mu}$ (i.e., shifted by $Q_{\mu}$ ) as functions of $k$. Dashed lines denote the corresponding time-averaged KHM contributions (see Figs. 6 and 7): blue, $-\langle\partial \mathcal{S} / \partial t\rangle_{t} / 4-Q_{\mu}$ (i.e., shifted by $Q_{\mu}$ ); green, $\mathcal{K}$; orange, $\left\langle\Psi^{\prime}\right\rangle_{t}$; and red, $-\left\langle\mathcal{D}^{\prime}\right\rangle_{t}$ as functions of $l=$ $\sqrt{3} / k$. All the quantities are normalized with respect to $Q_{\mu}$.

obtained from the ST and KHM relations are comparable $\left\langle S_{k}\right\rangle_{t} \simeq\langle\mathcal{K}\rangle_{t}$; the same is true for the pressure-dilatation induced cross-scale transfers $\left\langle\Psi_{k}\right\rangle_{t} \simeq-\langle\Psi\rangle_{t}$.

The decay and dissipation terms have comparable behaviors when shifted by the dissipation rate $Q_{\mu}$. This may be expressed as (here we leave out the time averages)

$$
\frac{\partial E_{\mathrm{k} k}}{\partial t}+\frac{1}{4} \frac{\partial \mathcal{S}}{\partial t} \simeq-Q_{\mu}, \quad D_{k}+\mathcal{D}^{\prime} \simeq Q_{\mu} .
$$

The ST and KHM quantities are complementary, as expected. As $\partial E_{\mathrm{k} k} / \partial t$ represents the rate of change of the kinetic energy on scales with wave-vector magnitudes smaller than or equal to $k, \partial \mathcal{S} / \partial t / 4$ gives approximatively the remaining decay rate (for wave-vector magnitudes larger than $k$ ). Similarly, $D_{k}$ is the dissipation rate on the scales $\leqslant k$ whereas $\mathcal{D}^{\prime}$ represents about the complementary dissipation rate (on the scales $>k$ ).

\section{INTERNAL ENERGY}

In the previous section, we showed that the exchanges between the kinetic and internal energies lead to a transfer of kinetic energy from large to small scales. It is interesting, therefore, to look at the scale dependence of the internal energy and its cross-scale transfer. 


\section{A. Spectral transfer}

One possible description of the spectral scale decomposition and cross-scale transfer of the internal energy could be done through the variable $q=(\gamma \rho T)^{1 / 2}$ [17]. Its evolution, following from Eq. (3), is given by

$$
\begin{aligned}
\frac{\partial q}{\partial t}+(\boldsymbol{u} \cdot \nabla) q+\frac{1}{2} q \theta= & \frac{\alpha \gamma}{2} \frac{1}{q} \Delta T-\frac{1}{2} \gamma(\gamma-1) \frac{1}{q} p \theta \\
& +\frac{1}{2} \gamma(\gamma-1) \frac{1}{q} \boldsymbol{\Sigma}: \boldsymbol{\tau} .
\end{aligned}
$$

We set the spectral decomposition of the internal energy, analogously to the case of the kinetic one, as a low-pass filtered quantity

$$
E_{\mathrm{i} k}=\frac{1}{\gamma(\gamma-1)} \sum_{\left|\boldsymbol{k}^{\prime}\right| \leqslant k}|\widehat{q}|^{2} .
$$

For $E_{\mathrm{i} k}$, one gets the following dynamic equation:

$$
\frac{\partial E_{\mathrm{i} k}}{\partial t}+S_{\mathrm{i} k}=\Phi_{\mathrm{i} k}-\Psi_{\mathrm{i} k}+D_{\mathrm{i} k}
$$

where

$$
\begin{aligned}
S_{\mathrm{i} k} & =\frac{1}{\gamma(\gamma-1)} \operatorname{Re} \sum_{\left|\boldsymbol{k}^{\prime}\right| \leqslant k}\left[2 \widehat{q}^{*}\left(\widehat{\boldsymbol{u} \cdot \boldsymbol{\nabla})} q+\widehat{q}^{*} \widehat{q \theta}\right],\right. \\
\Psi_{\mathrm{i} k} & =\frac{1}{\gamma} \operatorname{Re} \sum_{\left|\boldsymbol{k}^{\prime}\right| \leqslant k} \widehat{q}^{*} \widehat{q \theta}, \quad D_{\mathrm{i} k}=\operatorname{Re} \sum_{\left|\boldsymbol{k}^{\prime}\right| \leqslant k} \widehat{q}^{*} \widehat{q^{-1} \boldsymbol{\Sigma}: \boldsymbol{\tau}}, \\
\Phi_{\mathrm{i} k} & =\frac{\alpha}{(\gamma-1)} \operatorname{Re} \sum_{\left|\boldsymbol{k}^{\prime}\right| \leqslant k} \widehat{q}^{*} \widehat{q^{-1} \Delta T} .
\end{aligned}
$$

Here $S_{\mathrm{i} k}$ describes the cross-scale energy transfer, $\Phi_{\mathrm{i} k}$ results from the thermal diffusion, $\Psi_{\mathrm{i} k}$ is a term representing the pressure-dilatation effect, and $D_{\mathrm{i} k}$ comes from the viscous heating.

As there is no clear pressure-dilation-induced cross-scale transfer in run 1, we look only at run 2. We define the validity test of Eq. (42) as before by

$$
O_{\mathrm{i} k}=-\frac{\partial E_{\mathrm{i} k}}{\partial t}-S_{\mathrm{i} k}+\Phi_{\mathrm{i} k}-\Psi_{\mathrm{i} k}+D_{\mathrm{i} k}
$$

Figure 9(a) displays $O_{\mathrm{i} k}$ and its constituents, obtained at $t_{\omega}$ and $t_{\omega}+\Delta t$. Equation (42) is well satisfied, $\left|O_{\mathrm{i} k}\right| / Q_{\mu}<0.007 . \partial E_{\mathrm{i} k} / \partial t$ is positive as the internal energy increases and varies mostly on large scales. $D_{\mathrm{i} k}$ is about constant, $\sim Q_{\mu}$. This is due to the fact that the nonlinear term $\boldsymbol{\tau}: \boldsymbol{\Sigma}$ heats everywhere in the simulation box and importantly contributes to the $k=0$ term. The pressuredilatation term varies on large scales, while the diffusion and the transfer term lead to weak scale redistribution of the internal energy.

Figure 9(b) shows the spectral transfer results averaged over one pressure-dilatation oscillation period, the mean values of the different terms, and their minimum and maximum values. The dissipation $\left\langle D_{\mathrm{i} k}\right\rangle_{t} \simeq Q_{\mu}$ with weak variations and $\left\langle\partial E_{\mathrm{i} k} / \partial t\right\rangle_{t} \simeq Q_{\mu}$ with large temporal variations. The pressure dilatation $\left\langle\Psi_{\mathrm{i} k}\right\rangle_{t}$ is small and negative (with large fluctuations). The diffusion $\left\langle\Psi_{\mathrm{i} k}\right\rangle_{t}$ is weak with positive values, and the cross-scale transfer $\left\langle S_{\mathrm{i} k}\right\rangle_{t}$ is small with large fluctuations of large scales. In analogy with the spectral analysis for the kinetic energy, the nonlinear term $S_{\mathrm{i} k}$ leads to transfer of the internal energy from large to small scales, whereas the diffusion and the pressure dilatation lead to transfer of the internal energy in the opposite direction. These processes roughly compensate each other, and the dominant energy channel is the viscous heating $\left\langle\partial E_{\mathrm{i} k} / \partial t\right\rangle_{t} \simeq\left\langle D_{\mathrm{i} k}\right\rangle_{t}$. It is also clear that the dynamic spectral description of the internal energy, Eq. (42), is hardly 


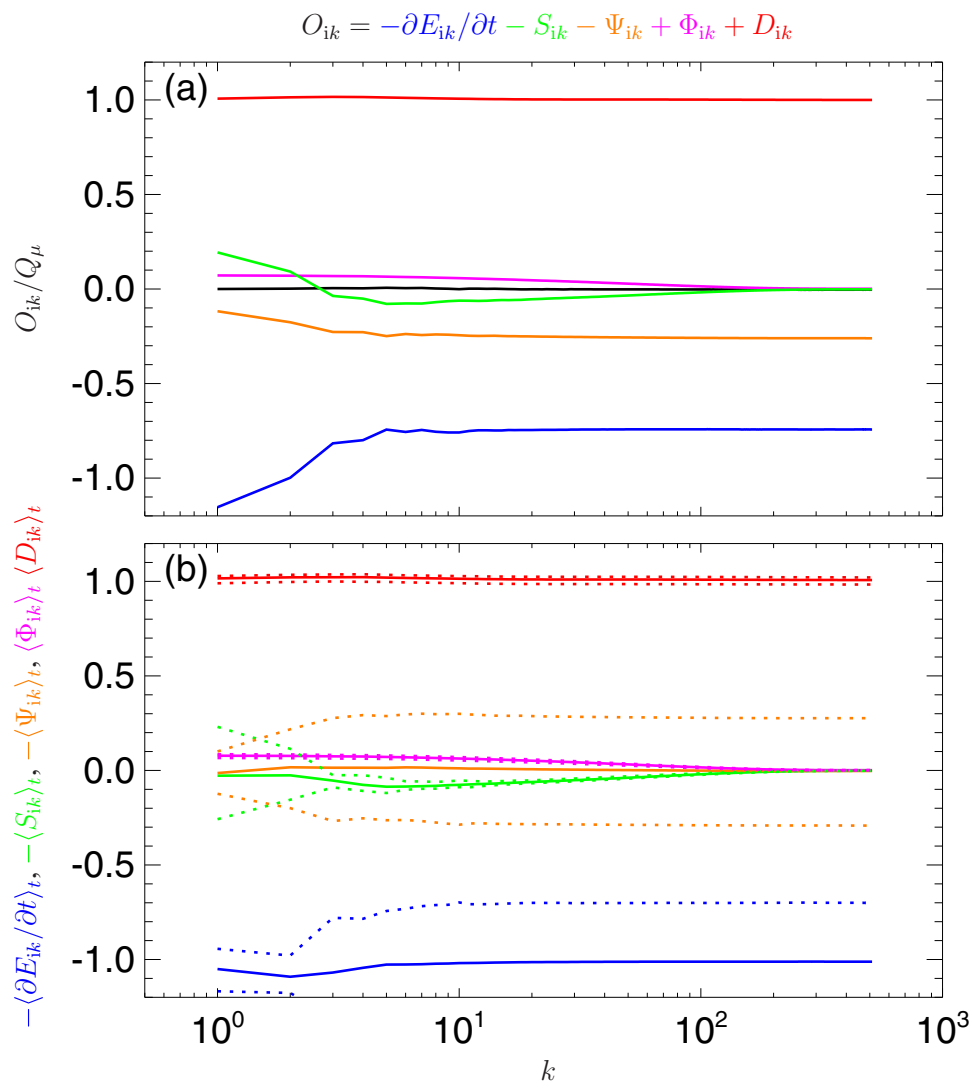

FIG. 9. Spectral transfer of the internal energy in run 2: (a) The validity test $O_{\mathrm{ik}}$ of Eq. (44) (black line) as a function of $k$ along with the different contributions: blue, the time variation $-\partial E_{\mathrm{i} k} / \partial t$; green, the energy transfer term $-S_{\mathrm{i} k}$; orange, the pressure-dilatation term $\Psi_{\mathrm{i} k}$; magenta, the diffusion $\Phi_{\mathrm{i} k}$; and red, the dissipation term $D_{\mathrm{i} k}$. (b) Time-averaged contributing terms (solid lines) with their minimum and maximum values (dotted lines) for blue, the decay $-\left\langle\partial E_{\mathrm{i} k} / \partial t\right\rangle_{t}$; green, the transfer $-\left\langle S_{\mathrm{i} k}\right\rangle_{t}$; orange, the pressure-dilatation term $\left\langle\Psi_{\mathrm{i} k}\right\rangle_{t}$; magenta, the diffusion $\left\langle\Phi_{\mathrm{i} k}\right\rangle_{t}$; and red, the dissipation term $\left\langle D_{\mathrm{i} k}\right\rangle_{t}$. All the quantities are normalized with respect to $Q_{\mu}$.

comparable to that for the kinetic energy, Eq. (18), especially concerning the viscous dissipation; compare Figs. 5 and 9. On the other hand, the pressure-dilatation terms for the kinetic [Eq. (18)] and internal [Eq. (42)] energies are comparable, $\Psi_{\mathrm{i} k} \simeq \Psi_{k}$. For the combined quantity $E_{\mathrm{k} k}+E_{\mathrm{i} k}$, the pressure-dilatation terms cancel each other, as one may expect. On the other hand, the dissipation terms have very different scale representations, so that $E_{\mathrm{k} k}+E_{\mathrm{i} k}$ clearly does not represent the total energy; the kinetic and internal energies ought to be treated separately.

\section{B. KHM equation}

One way to represent the internal energy in the KHM approach is the structure function [21]

$$
\mathcal{S}_{\mathrm{i}}=\langle\delta \rho \delta e\rangle .
$$

From Eq. (3), it follows for $e$ that

$$
\rho \frac{\partial e}{\partial t}+\rho(\boldsymbol{u} \cdot \nabla) e=\alpha \Delta e-p \theta+\boldsymbol{\tau}: \boldsymbol{\Sigma}
$$


and for $\mathcal{S}_{\mathrm{i}}$ one gets the dynamic KHM-like equation

$$
\frac{\partial \mathcal{S}_{\mathrm{i}}}{\partial t}+\nabla \cdot \mathcal{Y}_{\mathrm{i}}+\mathcal{R}_{\mathrm{i}}=2 \Phi_{\mathrm{i}}-2 \Psi_{\mathrm{i}}+2 \mathcal{D}_{\mathrm{i}}
$$

where

$$
\begin{aligned}
& \mathcal{Y}_{\mathrm{i}}=\langle\delta \boldsymbol{u} \delta \rho \delta e\rangle, \quad \mathcal{R}_{\mathrm{i}}=\left\langle\left(\rho \theta^{\prime}-\rho^{\prime} \theta\right) \delta e\right\rangle, \\
& \Psi_{\mathrm{i}}=\mathcal{V}_{\rho}(p \theta) / 2, \quad \mathcal{D}_{\mathrm{i}}=\mathcal{V}_{\rho}(\boldsymbol{\tau}: \boldsymbol{\Sigma}) / 2, \\
& \Phi_{\mathrm{i}}=\alpha\left\langle\delta \rho \delta\left(\rho^{-1} \Delta e\right)\right\rangle / 2,
\end{aligned}
$$

and

$$
\mathcal{V}_{\rho}(a)=\left\langle\left(1-\frac{\rho}{\rho^{\prime}}\right) a^{\prime}+\left(1-\frac{\rho^{\prime}}{\rho}\right) a\right\rangle .
$$

In Eq. (47), $\mathcal{K}_{\mathrm{i}}=-\left(\nabla \cdot \mathcal{Y}_{\mathrm{i}}+\mathcal{R}_{\mathrm{i}}\right) / 2$ represents the cross-scale transfer connected with $\mathcal{S}_{\mathrm{i}}$.

The pressure-dilatation $\Psi_{\mathrm{i}}$ and the dissipation $\mathcal{D}_{\mathrm{i}}$ terms depend on the density variation; for a constant $\rho$, these terms disappear. This is the first indication that $\mathcal{S}_{\mathrm{i}}$ does not represent the internal energy in a way comparable to the kinetic energy structure function $\mathcal{S}$.

To test Eq. (47) on the simulation results of run 2, we define the departure as

$$
O_{\mathrm{i}}(l)=-\frac{1}{2} \frac{\partial \mathcal{S}_{\mathrm{i}}}{\partial t}+\mathcal{K}_{\mathrm{i}}+\Phi_{\mathrm{i}}-\Psi_{\mathrm{i}}+D_{\mathrm{i}}
$$

Figure 10(a) shows the departure (black) $O_{\mathrm{i}}$ as a function of the scale $l$ along with the different contributions, the decay term (blue) $-\partial \mathcal{S}_{\mathrm{i}} / \partial t / 2$, the energy transfer term (green) $\mathcal{K}_{\mathrm{i}}$, the pressure dilatation term (orange) $-\Psi_{\mathrm{i}}$, the dissipation term (red) $\mathcal{D}_{\mathrm{i}}$, and the diffusion term (magenta) $\Phi_{\mathrm{i}}$. The calculation is done on a subgrid of $256^{3}$. Equation (47) is well satisfied, $\left|\mathcal{S}_{\mathrm{i}}\right| / Q_{\mu}<0.005$.

The pressure-dilatation structure function terms for the kinetic [Eq. (31)] and internal [Eq. (47)] energies are similar, $\Phi_{\mathrm{i}} \simeq \Phi$. The diffusion term is small and (except for the sign) corresponds to the diffusion term in the ST approach [Eq. (42)]. The dissipation term is small with respect to the dissipation rate $Q_{\mu}$, indicating that the viscous heating is not well represented in Eq. (47). Consequently, the structure function $\mathcal{S}_{\mathrm{i}}=\langle\delta \rho \delta e\rangle$ decreases with time, in contrast with the internal energy $E_{\mathrm{i}}=\langle\rho e\rangle$, which increases (see Fig. 2). These properties remain unchanged even after averaging over one pressure-dilatation oscillation period, as displayed in Fig. 10(b). All the terms (except for the dissipation and diffusion ones) exhibit large variations, dominantly on the large scales. The averaged pressure-dilatation term is small and corresponds to that of the kinetic energy, $\left\langle\Phi_{\mathrm{i}}\right\rangle_{t} \simeq\langle\Phi\rangle_{t}$.

Combining Eq. (31) with Eq. (47) as $\partial\left(\mathcal{S} / 2+\mathcal{S}_{\mathrm{i}}\right) / \partial t$ one recovers to a large extent the results of Ref. [21] (note, however, that the pressure-dilatation effects are transformed in Ref. [21] to a contribution to the cascade term using the isothermal closure). For the combined quantity $\mathcal{S} / 2+\mathcal{S}$, the pressure-dilatation terms cancel each other, similar to the ST case. However, the scale dependence of the viscous dissipation/heating is significantly different in the two approaches, so that it is hard to interpret $\mathcal{S} / 2+\mathcal{S}_{\mathrm{i}}$ as a representative of the total energy. It would be better to investigate the kinetic and internal energies separately by Eqs. (31) and (47).

The choice $\delta \rho \delta e$ does not correspond to the ST equation in the previous section based on $|\widehat{q}|^{2}$ [17]. To get an alternative version of the internal energy KHM equation corresponding to Eq. (42), one can investigate $|\delta q|^{2}$. The resulting equation can be expressed in a form similar to Eq. (47) as

$$
\frac{\partial \mathcal{S}_{\mathrm{i} q}}{\partial t}+\nabla \cdot \mathcal{Y}_{\mathrm{i} q}+\mathcal{R}_{\mathrm{i} q}=\Phi_{\mathrm{i} q}-\Psi_{\mathrm{i} q}+\mathcal{D}_{\mathrm{i} q}
$$




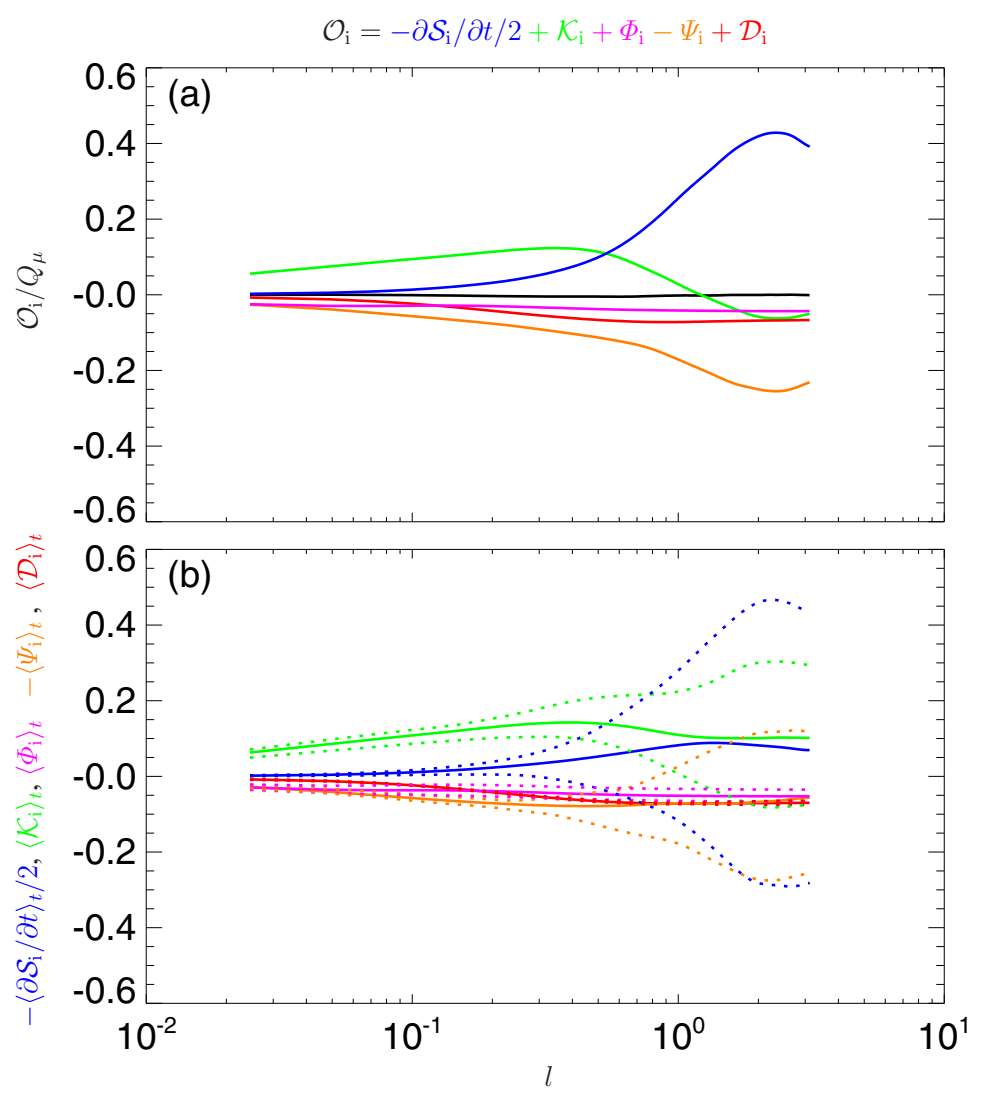

FIG. 10. KHM equation for the internal energy in run 2: (a) The validity test $O_{\mathrm{i}}$ of Eq. (49) (black line) as a function of the separation scale $l$ along with the different contributions: blue, $-\partial \mathcal{S}_{\mathrm{i}} / \partial t / 2$; green, $\mathcal{K}_{\mathrm{i}}$; orange, $-\Psi_{\mathrm{i}}$; red, $\mathcal{D}_{\mathrm{i}}$; and magenta, $\Phi_{\mathrm{i}}$. (b) Time-averaged contributing terms (solid lines) with their minimum and maximum values (dotted lines) for blue, $-\left\langle\partial \mathcal{S}_{\mathrm{i}} / \partial t\right\rangle_{t} / 2$; green, $\left\langle\mathcal{K}_{\mathrm{i}}\right\rangle_{t}$; orange, $-\left\langle\Psi_{\mathrm{i}}\right\rangle_{t}$; red, $\left\langle\mathcal{D}_{\mathrm{i}}\right\rangle_{t}$; and magenta, $\left\langle\Phi_{\mathrm{i}}\right\rangle_{t}$. All the quantities are normalized with respect to $Q_{\mu}$.

where

$$
\begin{aligned}
\mathcal{S}_{\mathrm{i} q} & =\frac{1}{\gamma(\gamma-1)}\left\langle|\delta q|^{2}\right\rangle, \quad \mathcal{Y}_{\mathrm{i} q}=\frac{1}{\gamma(\gamma-1)}\left\langle\delta \boldsymbol{u}|\delta q|^{2}\right\rangle, \\
\mathcal{R}_{\mathrm{i} q} & =\frac{1}{\gamma(\gamma-1)}\left\langle\delta q\left(q \theta^{\prime}-q^{\prime} \theta\right)\right\rangle, \\
\Psi_{\mathrm{i} q} & =\left\langle\delta q \delta\left(q^{-1} p \theta\right)\right\rangle, \quad \mathcal{D}_{\mathrm{i}}=\left\langle\delta q \delta\left(q^{-1} \boldsymbol{\Sigma}: \boldsymbol{\tau}\right)\right\rangle, \\
\Phi_{\mathrm{i} q} & =\frac{\alpha}{(\gamma-1)}\left\langle\delta q \delta\left(q^{-1} \Delta T\right)\right\rangle .
\end{aligned}
$$

Analyzing run 2 using this form of the internal energy KHM equation, we obtain results similar to those in Fig. 10. Therefore, also Eq. (50) should be investigated separately from Eq. (47).

\section{DISCUSSION}

In this paper, we investigated the properties of the spectral/spatial-scale distribution and the cross-scale transfer of the kinetic energy in compressible hydrodynamic turbulence. We used the 
dynamic spectral transfer (ST) Kármán-Howarth-Monin (KHM) equations, in compressible and incompressible forms, to analyze the results of two 3D direct numerical simulations of decaying compressible turbulence simulation with moderate Reynolds numbers and the initial Mach numbers $M=1 / 3$ and 1 . The simulations are initiated with large-scale solenoidal velocity fluctuations. The nonlinear coupling leads to a flux of the kinetic energy to small scales where it is dissipated; at the same time, the reversible pressure-dilatation mechanism causes oscillatory exchanges between the kinetic and internal energies with an average zero net energy transfer. While the simulations do not exhibit a clear inertial range, due largely to moderate Reynolds numbers, the dynamic compressible KHM and ST equations are well satisfied in the simulations. These approaches describe, in a quantitatively similar way for both methods, the decay of the kinetic energy on large scales, the energy transfer/cascade, the pressure dilatation, and the dissipation process. The incompressible versions are not valid, especially in run 2 (starting with $M=1$ ).

The ST approach that uses a low-pass filter in the $k$ space is by construction cumulative; in particular, the dissipation ST term reaches its (absolute) maximum values (given by the dissipation rate) at large $k$ (small scales). The KHM approach is complementary and has similar cumulative properties but in the opposite direction: the dissipation KHM term reaches its (absolute) maximum values at large scales (given as well by the dissipation rate). The comparison between the two approaches demonstrates that the range of scales where the dissipation is important is determined by the variations/gradients of the ST and KHM dissipation terms rather than their values. The same applies to the pressure dilatation: the pressure-dilatation terms in the ST and KHM exhibit opposite cumulative properties; they reach the average pressure dilatation at small and large scales, respectively. These results indicate that analyses based on the values of the cumulative pressure-dilatation terms are not very relevant; it is the variation over scales that counts. The pressure-dilatation energy exchange between the kinetic and internal energy becomes negligible when averaged over a period of pressure-dilatation oscillations. The time-averaged pressure dilatation may lead to a transfer of the kinetic energy from large to small scales (in agreement with Ref. [20]). For much larger systems, we expect that the pressure-dilatation energy exchange becomes negligible for any given time. This may explain the apparent discrepancy between the results of Refs. [12,19] and [20].

The results of both simulations indicate a simple relationship between the KHM and ST results through the inverse proportionality between the wave vector $k$ and the spatial separation length $l$ as $k l \simeq \sqrt{3}$, and they suggest a complementary scale-distribution meaning of the ST and KHM quantities. Interestingly, preliminary results of a similar comparison in two-dimensional Hall MHD simulations suggest a similar dependence $k l \simeq \sqrt{2}$ indicating that the relationship between the two scales depends on the space dimension. The simple relationship is useful to interpret the KMH results in the context of spectral analyses.

The ST approach is straightforward, requires fewer computational resources, and is directly linked to the spectral properties of velocity fluctuations. The KHM is more computationally demanding but leads to the so called exact scaling laws, and it can be directly used to analyze anisotropic turbulence [23,33]. We obtained similar results from the coarse-graining approach [34]. The coarse-graining approach presents semiquantitatively similar results concerning the energytransfer/cascade, decay, dissipation, and the pressure-dilatation processes; the localization of these different processes is, however, somewhat different when expressed in space-filtering scales with respect to the spatial separation scale. The cumulative features of the coarse-graining approach are similar to that of the KHM equation by construction (spatial low-pass filter), but a more detailed comparison between the coarse-graining method and the ST and KHM ones is beyond the scope of this paper.

We also investigated the properties of the internal energy using dynamic ST and KHM equations. These equations are well satisfied in both the simulations, and the descriptions of the pressuredilation effect are compatible with their counterpart for the kinetic energy. The ST and KHM equations for the kinetic and internal energies behave, however, very differently with respect to the viscous dissipation. Consequently, the ST and KHM (and likely also coarse-graining) approaches should better be used for the kinetic and internal energies separately. Moreover, the 
pressure-dilatation reversible coupling does not appear to lead to a net energy transfer between the kinetic and internal energies, at least in weakly compressible systems. It is, therefore, not necessary to investigate the two energies combined. The usage of combined quantities $[21,22]$ may lead to questionable results. For instance, in order to determine the heating rates of the turbulent cascade, it is necessary to look at the behavior of the kinetic energy (plus the magnetic energy in the magnetohydrodynamic case); the cascade/cross-scale transfer of the internal energy just leads to its redistribution.

Reference [19] analyzed the pressure-dilatation effect and showed that it decreases rapidly from large to small scales so that for a large enough system there are scales where the pressure dilatation becomes negligible and where the kinetic energy cascades in a conservative manner due to the nonlinear advection term. Our results further suggest that on larger scales, the kinetic energy is also conservatively transferred from large to small scales, partly due to the standard nonlinearadvection cascade and partly to the pressure-dilatation-induced energy transfer (the locality of the latter process is unclear). Our simulation results are limited by moderate Reynolds numbers and weak compressibilities, so they need to be extended to larger Reynolds number and higher Mach numbers [14,31,35-37].

\section{ACKNOWLEDGMENT}

P.H. acknowledges Grant No. 18-08861S of the Czech Science Foundation. L.F. is supported by the UK Science and Technology Facilities Council (STFC) Grant No. ST/T00018X/1.

[1] U. Frisch, Turbulence (Cambridge University Press, Cambridge, 1995).

[2] S. B. Pope, Turbulent Flows (Cambridge University Press, Cambridge, 2000).

[3] Y.-H. Pao, Structure of turbulent velocity and scalar fields at large wavenumbers, Phys. Fluids 8, 1063 (1965).

[4] P. D. Mininni, Scale interactions in magnetohydrodynamic turbulence, Annu. Rev. Fluid Mech. 43, 377 (2011).

[5] A. Alexakis, P. D. Mininni, and A. Pouquet, Shell-to-shell energy transfer in magnetohydrodynamics. I. Steady state turbulence, Phys. Rev. E 72, 046301 (2005).

[6] T. de Kármán and L. Howarth, On the statistical theory of isotropic turbulence, Proc. R. Soc. London A 164, 192 (1938).

[7] A. S. Monin and A. M. Yaglom, Statistical Fluid Mechanics: Mechanics of Turbulence (MIT Press, Cambridge, MA, 1975).

[8] A. N. Kolmogorov, Dissipation of energy in locally isotropic turbulence, Akad. Nauk SSSR Dokl. 32, 16 (1941).

[9] M. Germano, Turbulence: The filtering approach, J. Fluid Mech. 238, 325 (1992).

[10] G. L. Eyink and H. Aluie, Localness of energy cascade in hydrodynamic turbulence. I. Smooth coarse graining, Phys. Fluids 21, 115107 (2009).

[11] L. F. Richardson, Weather Prediction by Numerical Process (Cambridge University Press, Cambridge, 1922).

[12] D. S. Praturi and S. S. Girimaji, Effect of pressure-dilatation on energy spectrum evolution in compressible turbulence, Phys. Fluids 31, 055114 (2019).

[13] F. Bataille and Y. Zhou, Nature of the energy transfer process in compressible turbulence, Phys. Rev. E 59, 5417 (1999).

[14] G. L. Eyink and T. D. Drivas, Cascades and Dissipative Anomalies in Compressible Fluid Turbulence, Phys. Rev. X 8, 011022 (2018).

[15] H. Aluie, Scale decomposition in compressible turbulence, Physica D 247, 54 (2013).

[16] C. C. K. Lai, J. J. Charonko, and K. Prestridge, A Kármán-Howarth-Monin equation for variable-density turbulence, J. Fluid Mech. 843, 382 (2018). 
[17] W. Schmidt and P. Grete, Kinetic and internal energy transfer in implicit large-eddy simulations of forced compressible turbulence, Phys. Rev. E 100, 043116 (2019).

[18] H. Aluie, Compressible Turbulence: The Cascade and Its Locality, Phys. Rev. Lett. 106, 174502 (2011).

[19] H. Aluie, S. Li, and H. Li, Conservative cascade of kinetic energy in compressible turbulence, Astrophys. J. Lett. 751, L29 (2012).

[20] J. Wang, M. Wan, S. Chen, and S. Chen, Kinetic energy transfer in compressible isotropic turbulence, J. Fluid Mech. 841, 581 (2018).

[21] S. Galtier and S. Banerjee, Exact Relation for Correlation Functions in Compressible Isothermal Turbulence, Phys. Rev. Lett. 107, 134501 (2011).

[22] S. Banerjee and S. Galtier, A Kolmogorov-like exact relation for compressible polytropic turbulence, J. Fluid Mech. 742, 230 (2014).

[23] A. Verdini, R. Grappin, P. Hellinger, S. Landi, and W. C. Müller, Anisotropy of third-order structure functions in MHD turbulence, Astrophys. J. 804, 119 (2015).

[24] D. Pekurovsky, P3DFFT: A framework for parallel computations of Fourier transforms in three dimensions, SIAM J. Sci. Comput. 34, C192 (2012).

[25] M. Frigo and S. G. Johnson, The design and implementation of FFTW3, Proc. IEEE 93, 216 (2005).

[26] S. Kida and S. A. Orszag, Energy and spectral dynamics in decaying compressible turbulence, J. Sci. Comput. 7, 1 (1992).

[27] A. Pouquet, E. Lee, M. E. Brachet, P. D. Mininni, and D. Rosenberg, The dynamics of unforced turbulence at high Reynolds number for Taylor-Green vortices generalized to MHD, Geophys. Astrophys. Fluid Dyn. 104, 115 (2010).

[28] T. Passot and A. Pouquet, Numerical simulation of compressible homogeneous flows in the turbulent regime, J. Fluid Mech. 181, 441 (1987).

[29] S. Kida and S. A. Orszag, Energy and spectral dynamics in forced compressible turbulence, J. Sci. Comput. 5, 85 (1990).

[30] T. Gotoh, D. Fukayama, and T. Nakano, Velocity field statistics in homogeneous steady turbulence obtained using a high-resolution direct numerical simulation, Phys. Fluids 14, 1065 (2002).

[31] T. Ishihara, T. Gotoh, and Y. Kaneda, Study of high-Reynolds number isotropic turbulence by direct numerical simulation, Annu. Rev. Fluid Mech. 41, 165 (2009).

[32] J. P. Graham, R. Cameron, and M. Schüssler, Turbulent small-scale dynamo action in solar surface simulations, Astrophys. J. 714, 1606 (2010).

[33] C. Cambon, L. Danaila, F. S. Godeferd, and J. F. Scott, Third-order statistics and the dynamics of strongly anisotropic turbulent flows, J. Turbul. 14, 121 (2013).

[34] P. Hellinger, A. Verdini, S. Landi, L. Franci, E. Papini, and L. Matteini, On cascade of kinetic energy in compressible hydrodynamic turbulence, arXiv:2004.02726.

[35] T. D. Drivas and G. L. Eyink, An Onsager singularity theorem for turbulent solutions of compressible Euler equations, Commun. Math. Phys. 359, 733 (2018).

[36] A. G. Kritsuk, R. Wagner, and M. L. Norman, Energy cascade and scaling in supersonic isothermal turbulence, J. Fluid Mech. 729, R1 (2013).

[37] R. Ferrand, S. Galtier, F. Sahraoui, and C. Federrath, Compressible turbulence in the interstellar medium: New insights from a high-resolution supersonic turbulence simulation, Astrophys. J. 904, 160 (2020). 